Heravi, M, Marin, L and Sebu, C

The method of fundamental solutions for complex electrical impedance tomography

Heravi, M, Marin, L and Sebu, C (2014) The method of fundamental solutions for complex electrical impedance tomography. Engineering Analysis with Boundary Elements, 46. pp. 126-139.

doi: 10.1016/j.enganabound.2014.04.022

This version is available: https://radar.brookes.ac.uk/radar/items/6b4b2cfa-8f67-42cf-bf0c-9852e1054a20/1/

Available on RADAR: July 2016

Copyright $(C$ and Moral Rights are retained by the author(s) and/ or other copyright owners. A copy can be downloaded for personal non-commercial research or study, without prior permission or charge. This item cannot be reproduced or quoted extensively from without first obtaining permission in writing from the copyright holder(s). The content must not be changed in any way or sold commercially in any format or medium without the formal permission of the copyright holders.

This document is the post print version of the journal article. Some differences between the published version and this version may remain and you are advised to consult the published version if you wish to cite from it. 


\title{
THE METHOD OF FUNDAMENTAL SOLUTIONS FOR COMPLEX ELECTRICAL IMPEDANCE TOMOGRAPHY
}

\author{
Marjan Asadzadeh Heravi ${ }^{1, *}$, Liviu Marin ${ }^{2,3, \dagger}$, and Cristiana Sebu ${ }^{1, \ddagger}$ \\ ${ }^{1}$ Department of Mechanical Engineering and Mathematical Sciences, Oxford Brookes University, \\ Wheatley Campus, Oxford OX33 1HX, UK \\ ${ }^{2}$ Department of Mathematics, Faculty of Mathematics and Computer Science, University of Bucharest, \\ 14 Academiei, 010014 Bucharest, Romania \\ ${ }^{3}$ Institute of Solid Mechanics, Romanian Academy, 15 Constantin Mille, 010141 Bucharest, Romania
}

\begin{abstract}
The forward problem for complex electrical impedance tomography (EIT) is solved by means of a meshless method, namely the method of fundamental solutions (MFS). The MFS for the complex EIT direct problem is numerically implemented, and its efficiency and accuracy as well as the numerical convergence of the MFS solution are analysed when assuming the presence in the medium (i.e. background) of one or two inclusions with the physical properties different from those corresponding to the background. Four numerical examples with inclusion(s) of various convex and non-convex smooth shapes (e.g. circular, elliptic, peanut-shaped and acorn-shaped) and sizes are presented and thoroughly investigated.
\end{abstract}

Keywords: Electrical impedance tomography (EIT); Multi-frequency; Forward problem; Method of fundamental solutions (MFS); Meshless method.

\section{INTRODUCTION}

Electrical impedance tomography (EIT) is a technique used for determining the admittivity distribution in the interior of an object, given simultaneous measurements of alternating electric currents (of frequencies varying from $10 \mathrm{~Hz}-500 \mathrm{kHz}$ ) and of induced voltages on the boundary of the object [12]. For a given conductive object, the admittivity is a complex valued function whose real part is the electrical conductivity and whose imaginary part is the product of the frequency of the applied electric alternating current and the permittivity of the object. Since different materials display different electrical properties, a map of internal admittivity can be used to infer the internal structure of the object under consideration. Therefore, EIT can be used as a non-invasive and portable method of industrial, geophysical and medical imaging [1].

The reconstruction procedures proposed for static EIT include a wide range of iterative methods based on formulating the inverse problem in the framework of nonlinear optimization $[2,24,40,41]$. These approaches usually involve estimating the admittivity distribution of the object under consideration and then solve the forward problem (often using finite element methods [FEMs]) for the same input current patterns to compute the boundary voltages and then comparing the boundary data predicted by this estimate with the measured data. The discrepancy between these two data sets is then used to update the admittivity estimate and the procedure is repeated until a satisfactory agreement

\footnotetext{
${ }^{*}$ E-mail: marjan.asadzadeh.heravi-2012@brookes.ac.uk

${ }^{\dagger}$ Corresponding author. E-mails: marin.liviu@gmail.com; liviu.marin@fmi.unibuc.ro

${ }^{\ddagger}$ E-mail: csebu@brookes.ac.uk
} 
is achieved. However, the solution of the inverse problem in static EIT has suffered not only from its ill-posedness due to the inherent insensitivity of boundary measurements to any small changes of interior conductivity and permittivity values and its poor spatial resolution, but also from its reliance on accurate forward models which mimic every aspect of the imaging object (e.g. knowledge of boundary geometry, electrode positions and other sources of systematic artifacts in measured data). Hence, EIT has had limited applicability so far in clinical applications. This has encouraged the search of new reconstruction methods, such as the time-difference EIT (tdEIT) or frequency-difference EIT (fdEIT). Even though numerous tdEIT methods have been applied to image lung functions, stomach emptying or brain functions $[12,31,32]$, there are medical applications where these time-reference data is not available (e.g. breast cancer or cerebral stroke detection). Since complex conductivity spectra of biological tissues show frequency-dependent changes [8, 32], fdEIT methods have been proposed to image the changes in the admittivity distribution with respect to frequency $[11,13,22,38]$. It has been showed that, although there are no visible differences in the reconstructed frequency-difference images even though the true admittivity distributions have a strong frequency dependence, any anomaly can be clearly identified as long as its admittivity differs significantly from that of the background which is the case for tumour and stroke imaging.

To achieve clinical acceptance, the theoretical developments of EIT reconstruction methods need to be closely connected with laboratory experiments and studies on real data. This implies not only the modelling of the real geometry and data collection devices but also the development of a computer software fast enough to be used for real-time monitoring. However, optimized image reconstruction techniques for EIT rely on computationally efficient and numerically robust forward solvers. In this paper, we address this need by presenting an algorithm based on the method of fundamental solutions (MFS) which can be successfully used to find the numerical solution of the EIT forward problem for piecewise constant admittivity distributions to a high level of precision.

The MFS is a meshless boundary collocation method applicable to boundary value problems in which a fundamental solution of the operator in the governing equation is known explicitly. The basic ideas of this method were first introduced, in the early 60's, by Kupradze and Aleksidze [23], whilst its numerical formulation was first given by Mathon and Johnston [30] in the late 70's. The main idea of the MFS consists of approximating the solution of the problem by a linear combination of fundamental solutions with respect to some singularities/source points which are located outside the domain. Consequently, the original problem is reduced to determining both the unknown coefficients of the fundamental solutions and the coordinates of the source points by requiring the approximation to satisfy the boundary conditions in some sense and hence solving a non-linear problem. If the source points are fixed a priori then the coefficients of the MFS approximation are determined by solving a linear problem. The aforementioned MFS procedures are referred to in the literature as the dynamic and static approaches, respectively.

Despite its constraint on the knowledge of a fundamental solution of the governing partial differential equation, the MFS has become very popular especially due to the ease with which it can be implemented, in particular for problems in complex geometries. It should be mentioned that the MFS can be used in conjunction with the so-called singularity subtraction technique (SST) for problems with boundary and/or solution singularities, in the sense that the standard MFS solution of the partial differential equation investigated is augmented by suitable singular functions/eigenfunctions associated with the corresponding partial differential operator as given by the asymptotic expansion of the solution near the singular point. For details on the MFS-SST procedure applied to the numerical solution of direct and inverse problems associated with the Laplace, biharmonic, Helmholtz and modified Helmholtz, and Cauchy-Navier equations, we refer the reader to Karageorghis [14], Karageorghis et al. [21], and Marin [25-27]. In the MFS, there are no integrations. This clearly represents an advantage over the boundary element method (BEM) where integrations could be potentially troublesome and complicated. Furthermore, the MFS is a boundary collocation method, and hence only the boundary of the solution 
domain needs to be discretised, which is another advantage of the MFS over domain discretisation methods, such as FEMs or finite difference methods (FDMs). The major disadvantages of the MFS are related to the optimal location of the singularities, which is an important issue especially from the numerical point of view, and its inability to be directly applied to non-homogeneous or nonlinear partial differential equations, for which a fundamental solution is not available.

The MFS has been successfully applied to a large variety of physical problems, such as the Laplace equation [14, 25, 35, 37], the biharmonic equation [16, 34], the Helmholtz and modified Helmholtz equations [15, 26, 27], scattering and radiation problems [6], elastostatics [17, 19, 21, 36], thermoelasticity [28, 39], inhomogeneous elliptic equations [34], heat conduction problems in functionally graded materials [29], layered [3] and composite materials [4, 18], etc. For a detailed and comprehensive description of the application of the MFS to direct and inverse problems we refer the reader to the survey papers by Fairweather and Karageorghis [5], Fairweather et al. [6], Golberg and Chen [9], and Karageorghis et al. [20], as well as the references therein.

To the best of our knowledge, the application of the MFS to the forward problem for complex EIT has not been investigated yet. Motivated by the success of the application of the MFS to various physical problems, as well as the importance of the complex EIT forward problem, the purpose of the present study is to propose, implement and analyse the application of the MFS to the aforementioned problem. We also investigate the efficiency, accuracy and numerical convergence of the MFS solution of the complex EIT forward problem when assuming the presence in the medium (i.e. background) of one or two inclusions with physical properties (i.e. conductivity and permittivity) different from those of the background. The numerical analysis is studied for various convex and non-convex smooth shapes (e.g. circular, elliptic, peanut-shaped and acorn-shaped) and sizes of the inclusion(s). The paper is structured as follows. In Section 2 we formulate mathematically the problem investigated herein. The MFS application to the complex EIT forward problem is presented in Section 3. The efficiency, accuracy and numerical convergence of the proposed method are validated by considering four examples in Section 4. Finally, some conclusions and future related problems are presented in Section 5.

\section{Mathematical Formulation}

Let $\Omega$ be a bounded, simply connected domain in $\mathbb{R}^{2}$. The forward complex EIT problem can be stated as follows: for a given complex admittivity scalar-valued function $\gamma(\mathbf{x})=\sigma(\mathbf{x})+i \omega \varepsilon(\mathbf{x}), \mathbf{x} \in \bar{\Omega}$, with strictly positive real part $\sigma(\mathbf{x}) \geq c>0$, at a frequency $\omega$ of the applied current, find the complex potential distribution $\phi(\mathbf{x})=v(\mathbf{x})+\mathrm{i} h(\mathbf{x}), \mathbf{x} \in \bar{\Omega}$, satisfying the elliptic partial differential equation

$$
\nabla \cdot(\gamma(\mathbf{x}) \nabla \phi(\mathbf{x}))=0, \quad \mathbf{x} \in \Omega
$$

subject to the Neumann boundary condition

$$
\gamma(\mathbf{x})(\mathbf{n}(\mathbf{x}) \cdot \nabla \phi(\mathbf{x}))=I(\mathbf{x}, \omega), \quad \mathbf{x} \in \partial \Omega, \quad \text { such that } \int_{\partial \Omega} I(\mathbf{x}, \omega) \mathrm{d} \mathbf{x}=0,
$$

where $\mathbf{n}(\mathbf{x})$ is the outer normal at $\mathbf{x} \in \partial \Omega$.

If $\gamma \in \mathrm{L}^{\infty}(\bar{\Omega})$, the Neumann boundary value problem (1)-(2), for $I \in \mathrm{H}^{-\frac{1}{2}}(\partial \Omega)$, has a unique solution $\phi \in \mathrm{H}^{1}(\Omega)$ up to an additive constant [7], which we fix by choosing the ground as

$$
\int_{\partial \Omega} \phi(\mathbf{x}) \mathrm{d} \mathbf{x}=0
$$

In this paper, we are interested to find the numerical solution to the EIT forward problem for a piecewise constant admittivity distribution. To this end, we let $\Omega_{j}, j=\overline{1, J}$, be disconnected subdomains compactly contained in $\Omega$, such that $\Omega_{k} \cap \Omega_{l}=\varnothing$ for all $k, l=\overline{1, J}, k \neq l$, and $\Omega_{0}=\Omega \backslash \bigcup_{j=1}^{J} \Omega_{j}$ 
is connected, see Figure 1. The following notations are also introduced

$$
\begin{aligned}
& \Gamma_{0}=\partial \Omega_{0} \backslash \bigcup_{j=1}^{J} \partial \Omega_{j}, \\
& \Gamma_{j}=\partial \Omega_{j}, \quad j=\overline{1, J}, \\
& \phi_{j}(\mathbf{x})=v_{j}(\mathbf{x})+\mathrm{i} h_{j}(\mathbf{x}), \quad \mathbf{x} \in \bar{\Omega}_{j}=\Omega_{j} \cup \partial \Omega_{j}, \quad j=\overline{0, J}, \\
& \gamma_{j}=\sigma_{j}+\mathrm{i} \omega \varepsilon_{j}, \quad j=\overline{0, J},
\end{aligned}
$$

where both the conductivity $\sigma_{j}$ and the permittivity $\varepsilon_{j}$ distributions in $\bar{\Omega}_{j}$ are assumed to be constant. Thus, equation (1) reduces to a set of Laplace equations

$$
\nabla \cdot\left(\nabla \phi_{j}(\mathbf{x})\right)=0, \quad \mathbf{x} \in \Omega_{j}, \quad j=\overline{0, J},
$$

which could be solved subject to a Neumann boundary condition

$$
\gamma_{0}\left(\mathbf{n}^{(0)}(\mathbf{x}) \cdot \nabla \phi_{0}(\mathbf{x})\right)=I_{0}(\mathbf{x}) \mathrm{e}^{\mathrm{i} \omega t}, \quad \mathbf{x} \in \Gamma_{0}, \quad \text { such that } \int_{\Gamma_{0}} I_{0}(\mathbf{x}) \mathrm{d} \mathbf{x}=0,
$$

at a set frequency $\omega$ and time $t$. To guarantee uniqueness, we impose

$$
\int_{\Gamma_{0}} \phi_{0}(\mathbf{x}) \mathrm{d} \mathbf{x}=0
$$

Due to the continuity of the potential and the current flux densities across the interfaces $\Gamma_{j}$, the solutions $\phi_{j}, j=\overline{0, J}$, should also satisfy the following transmission conditions:

$$
\begin{aligned}
& \phi_{0}(\mathbf{x})-\phi_{j}(\mathbf{x})=0, \quad \mathbf{x} \in \Gamma_{j}, \quad j=\overline{1, J}, \\
& \gamma_{0}\left(\mathbf{n}^{(0)}(\mathbf{x}) \cdot \nabla \phi_{0}(\mathbf{x})\right)+\gamma_{j}\left(\mathbf{n}^{(j)}(\mathbf{x}) \cdot \nabla \phi_{j}(\mathbf{x})\right)=0, \quad \mathbf{x} \in \Gamma_{j}, \quad j=\overline{1, J},
\end{aligned}
$$

where $\mathbf{n}^{(j)}(\mathbf{x})$ are the outer normals at $\mathbf{x} \in \partial \Omega_{j}, j=\overline{0, J}$, see Figure 1 .

Equations (5a)-(5e) can be rewritten in terms of $v_{j}=\operatorname{Re} \phi_{j}$ and $h_{j}=\operatorname{Im} \phi_{j}, j=\overline{0, J}$, as follows:

$$
\begin{array}{ll}
\Delta v_{j}(\mathbf{x})=0, & \mathbf{x} \in \Omega_{j}, \quad j=\overline{0, J}, \\
\Delta h_{j}(\mathbf{x})=0, & \mathbf{x} \in \Omega_{j}, \quad j=\overline{0, J},
\end{array}
$$

subject to the Neumann boundary conditions

$$
\begin{array}{ll}
\mathbf{n}^{(0)}(\mathbf{x}) \cdot\left(\sigma_{0} \nabla v_{0}(\mathbf{x})-\omega \varepsilon_{0} \nabla h_{0}(\mathbf{x})\right)=I_{0}(\mathbf{x}) \cos (\omega t), & \mathbf{x} \in \Gamma_{0}, \\
\mathbf{n}^{(0)}(\mathbf{x}) \cdot\left(\omega \varepsilon_{0} \nabla v_{0}(\mathbf{x})+\sigma_{0} \nabla h_{0}(\mathbf{x})\right)=I_{0}(\mathbf{x}) \sin (\omega t), & \mathbf{x} \in \Gamma_{0},
\end{array}
$$

the uniqueness conditions

$$
\begin{aligned}
& \int_{\Gamma_{0}} v_{0}(\mathbf{x}) \mathrm{d} \mathbf{x}=0, \\
& \int_{\Gamma_{0}} h_{0}(\mathbf{x}) \mathrm{d} \mathbf{x}=0,
\end{aligned}
$$

and the transmission conditions

$$
\begin{aligned}
& v_{0}(\mathbf{x})-v_{j}(\mathbf{x})=0, \quad \mathbf{x} \in \Gamma_{j}, \quad j=\overline{1, J}, \\
& h_{0}(\mathbf{x})-h_{j}(\mathbf{x})=0, \quad \mathbf{x} \in \Gamma_{j}, \quad j=\overline{1, J}, \\
& \mathbf{n}^{(0)}(\mathbf{x}) \cdot\left(\sigma_{0} \nabla v_{0}(\mathbf{x})-\omega \varepsilon_{0} \nabla h_{0}(\mathbf{x})\right)+\mathbf{n}^{(j)}(\mathbf{x}) \cdot\left(\sigma_{j} \nabla v_{j}(\mathbf{x})-\omega \varepsilon_{j} \nabla h_{j}(\mathbf{x})\right)=0, \quad \mathbf{x} \in \Gamma_{j}, j=\overline{1, J}, \\
& \mathbf{n}^{(0)}(\mathbf{x}) \cdot\left(\omega \varepsilon_{0} \nabla v_{0}(\mathbf{x})+\sigma_{0} \nabla h_{0}(\mathbf{x})\right)+\mathbf{n}^{(j)}(\mathbf{x}) \cdot\left(\omega \varepsilon_{j} \nabla v_{j}(\mathbf{x})+\sigma_{j} \nabla h_{j}(\mathbf{x})\right)=0, \quad \mathbf{x} \in \Gamma_{j}, j=\overline{1, J} .
\end{aligned}
$$




\section{The Method of Fundamental Solutions}

The fundamental solution of the two-dimensional Laplace equation is given by, see e.g. [5]

$$
\mathrm{F}(\mathbf{x}, \xi)=-\frac{1}{2 \pi} \log \|\mathbf{x}-\xi\|, \quad \mathbf{x} \in \bar{\Omega}_{j}, \quad j=\overline{0, J},
$$

where $\mathbf{x}=\left(\mathrm{x}_{1}, \mathrm{x}_{2}\right)$ is a collocation point, $\xi=\left(\xi_{1}, \xi_{2}\right) \in \mathbb{R}^{2} \backslash \bar{\Omega}_{j}$ is a singularity or source point and $\|\mathbf{x}-\xi\|=\sqrt{\left(\mathrm{x}_{1}-\xi_{1}\right)^{2}+\left(\mathrm{x}_{2}-\xi_{2}\right)^{2}}$ is the Euclidean distance between the collocation point $\mathbf{x}$ and the singularity/source point $\xi$. In Figure 1, we present the possible positions of the source and collocation points for a simple geometry.

In the MFS, both the real $\left(v_{j}\right)$ and the imaginary $\left(h_{j}\right)$ potentials corresponding to the background $(j=0)$ and inclusions $(j=\overline{1, J})$ are approximated by a linear combination of fundamental solutions with respect to $\mathrm{N}_{0}=\mathrm{N}_{00}+\mathrm{N}_{01}+\ldots+\mathrm{N}_{0 J}$ singularities, $\left\{\boldsymbol{\xi}^{(0 ; n)}\right\}_{n=1}^{\mathrm{N}_{0}}$, and $\mathrm{N}_{j}$ singularities, $\left\{\boldsymbol{\xi}^{(j ; n)}\right\}_{n=1}^{\mathrm{N}_{j}}$, $\overline{1, J}$, respectively, in the form

$$
\begin{array}{ll}
v_{j}(\mathbf{x}) \approx \sum_{n=1}^{\mathrm{N}_{j}} \mathrm{c}^{(j ; n)} \mathrm{F}\left(\mathbf{x}, \xi^{(j ; n)}\right), & \mathbf{x} \in \bar{\Omega}_{j}, \quad j=\overline{0, J}, \\
h_{j}(\mathbf{x}) \approx \sum_{n=1}^{\mathrm{N}_{j}} \mathrm{~d}^{(j ; n)} \mathrm{F}\left(\mathbf{x}, \xi^{(j ; n)}\right), & \mathbf{x} \in \bar{\Omega}_{j}, \quad j=\overline{0, J},
\end{array}
$$

where $\mathbf{c}^{(j)}=\left[\mathrm{c}^{(j ; 1)}, \ldots, \mathrm{c}^{\left(j ; \mathrm{N}_{j}\right)}\right]^{\top} \in \mathbb{R}^{\mathrm{N}_{j}}, \mathbf{d}^{(j)}=\left[\mathrm{c}^{(j ; 1)}, \ldots, \mathrm{c}^{\left(j ; \mathrm{N}_{j}\right)}\right]^{\top} \in \mathbb{R}^{\mathrm{N}_{j}}$ and $\xi^{(j)} \in \mathbb{R}^{2 \mathrm{~N}_{j}}, j=\overline{1, J}$, is a vector containing the coordinates of the singularities. It should be noted that, in the case of the background $(j=0)$, the total number of singularities (source points) was taken to be $\mathrm{N}_{0}=$ $\mathrm{N}_{00}+\mathrm{N}_{01}+\ldots+\mathrm{N}_{0 J}$ since $\partial \Omega_{0}$ is the outer boundary of the background and $\Gamma_{j}, j=\overline{1, J}$, are the $J$ inner boundaries of the background (interfaces between the background and the inclusions), whilst $\mathrm{N}_{00}$ and $\mathrm{N}_{0 j}, j=\overline{1, J}$ are singularities corresponding to the outer and inner boundaries (interfaces), respectively.

From equations $(6 \mathrm{c}),(6 \mathrm{~d}),(6 \mathrm{i}),(6 \mathrm{j})$ and $(7)$, it follows that the corresponding normal derivatives of the real and imaginary parts of the potential on $\partial \Omega_{j}$ can be approximated by

$$
\begin{aligned}
& \left(\mathbf{n}^{(j)}(\mathbf{x}) \cdot \nabla v_{j}(\mathbf{x})\right) \approx \sum_{n=1}^{\mathrm{N}_{j}} \mathrm{c}^{(j ; n)}\left[\mathbf{n}^{(j)}(\mathbf{x}) \cdot \nabla_{\mathbf{x}} \mathrm{F}\left(\mathbf{x}, \xi^{(j ; n)}\right)\right], \quad \mathbf{x} \in \partial \Omega_{j}, \quad j=\overline{0, J} \\
& \left(\mathbf{n}^{(j)}(\mathbf{x}) \cdot \nabla h_{j}(\mathbf{x})\right) \approx \sum_{n=1}^{\mathrm{N}_{j}} \mathrm{~d}^{(j ; n)}\left[\mathbf{n}^{(j)}(\mathbf{x}) \cdot \nabla_{\mathbf{x}} \mathrm{F}\left(\mathbf{x}, \xi^{(j ; n)}\right)\right], \quad \mathbf{x} \in \partial \Omega_{j}, \quad j=\overline{0, J} .
\end{aligned}
$$

Next, we select $\mathrm{M}_{j}$ collocation points, $\left\{\mathbf{x}^{(j ; n)}\right\}_{n=1}^{\mathrm{M}_{j}}$, on each boundary $\Gamma_{j}, j=\overline{0, J}$, such that the collocation points on each interface $\Gamma_{j}, j=\overline{1, J}$, are the same for both the boundary associated with the background, $\partial \Omega_{0}$, and the boundary corresponding to the $j$ th inclusion, $\partial \Omega_{j}$, i.e. the following relations hold:

$$
\mathbf{x}^{\left(0 ; \sum_{\ell=0}^{j-1} \mathrm{M}_{\ell}+k\right)}=\mathbf{x}^{(j ; k)}, \quad k=\overline{1, \mathrm{M}_{j}}, \quad j=\overline{1, J} .
$$

Consequently, the total number of boundary collocation points employed in the present approach is $\mathrm{M}_{0}+\mathrm{M}_{1}+\cdots+\mathrm{M}_{J}$, i.e. the number of boundary collocation points associated with the background $\Omega_{0}$, namely $\left\{\mathbf{x}^{(0 ; n)}\right\}_{n=1}^{\mathrm{M}_{0}+\mathrm{M}_{1}+\ldots+\mathrm{M}_{J}}$. 
Then, the collocation of the uniqueness conditions (6e) and (6f) yields the following equations

$$
\begin{aligned}
& \sum_{n=1}^{\mathrm{N}_{0}} \mathrm{c}^{(0 ; n)}\left[\sum_{k=1}^{\mathrm{M}_{0}} \mathrm{~F}\left(\mathrm{x}^{(0 ; k)}, \xi^{(0 ; n)}\right)\right] \approx 0 \\
& \sum_{n=1}^{\mathrm{N}_{0}} \mathrm{~d}^{(0 ; n)}\left[\sum_{k=1}^{\mathrm{M}_{0}} \mathrm{~F}\left(\mathrm{x}^{(0 ; k)}, \xi^{(0 ; n)}\right)\right] \approx 0 .
\end{aligned}
$$

Finally, we collocate the Neumann boundary conditions (6c) and (6d), as well as the transmission conditions $(6 \mathrm{~g})-(6 \mathrm{j})$ to obtain the following system of linear equations for the unknown coefficients $\mathbf{c}^{(j)}, \mathbf{d}^{(j)} \in \mathbb{R}^{\mathrm{N}_{j}}, j=\overline{0, J}$, grouped in the vector $\mathbf{X} \in \mathbb{R}^{\mathrm{N}}, \mathrm{N}=\mathrm{N}_{0}+\mathrm{N}_{1}+\ldots+\mathrm{N}_{J}$ :

$$
\mathbf{A} \mathbf{X}=\mathbf{f}
$$

where $\mathbf{A} \in \mathbb{R}^{(\mathrm{M}+2) \times \mathrm{N}}$ is the corresponding MFS matrix whose elements are calculated from equations (6c)-(6j), respectively, while $\mathbf{f} \in \mathbb{R}^{\mathrm{M}+2}, \mathrm{M}=2 \mathrm{M}_{0}+4 \mathrm{M}_{1}+\cdots+4 \mathrm{M}_{J}$, contains the corresponding discretised Neumann data from equations (6c) and (6d), as well as zero entries associated with the transmission conditions (6g)-(6j) and uniqueness conditions (11a) and (11b). Clearly, in order for the discretised MFS system of linear equations (10) to have a unique solution $\mathbf{X} \in \mathbb{R}^{\mathrm{N}}$, the numbers of collocation points $\mathrm{M}$ and singularities $\mathrm{N}$ should satisfy the inequality $\mathrm{N} \leq \mathrm{M}+2$. In this study, the system of linear equations (12) is solved in the least-squares sense by numerically inverting the normal system of equations associated with the system (12), namely

$$
\mathbf{X}=\left(\mathbf{A}^{\top} \mathbf{A}\right)^{-1}\left(\mathbf{A}^{\top} \mathbf{f}\right)
$$

\section{Numerical Results}

Without loss of generality, we assume that the background is described by the unit disk centered at the origin of the system of coordinates, i.e.

$$
\Omega=\left\{\mathrm{x} \in \mathbb{R}^{2} \mid \mathrm{x}_{1}^{2}+\mathrm{x}_{2}^{2}<R^{2}\right\}
$$

with $R=1$, and is characterised by the conductivity $\sigma_{0}=0.036 \mathrm{~S} \mathrm{~m}^{-1}$ and permittivity $\varepsilon_{0}=$ $1.9 \times 10^{-9} \mathrm{~F} \mathrm{~m}^{-1}$. In SI based units, $1 \mathrm{~S}=1 \mathrm{~A}^{2} \mathrm{~s}^{3} \mathrm{~m}^{-2} \mathrm{~kg}^{-1}$ and $1 \mathrm{~F}=1 \mathrm{~A}^{2} \mathrm{~s}^{4} \mathrm{~m}^{-2} \mathrm{~kg}^{-1}$. The inclusions considered in this study $\Omega_{j}, j=1,2$, are assumed to have smooth boundaries $\partial \Omega_{j}, j=1,2$, and be centered at $\mathbf{x}^{(1)}=(0.45,0)$ and $\mathbf{x}^{(2)}=(-0.45,0.35)$, respectively, whilst their corresponding conductivities and permittivities are given by $\sigma_{1}=0.042 \mathrm{~S} \mathrm{~m}^{-1}$ and $\varepsilon_{1}=9.7 \times 10^{-9} \mathrm{~F} \mathrm{~m}^{-1}$, and $\sigma_{2}=0.05 \mathrm{~S} \mathrm{~m}^{-1}$ and $\varepsilon_{2}=1.2 \times 10^{-8} \mathrm{~F} \mathrm{~m}^{-1}$, respectively.

Example 1. We consider the circular inclusion of radius $r=0.25$ and centered at $\mathbf{x}^{(1)}$, i.e.

$$
\Omega_{1}=\left\{\mathrm{x} \in \mathbb{R}^{2} \mid\left(\mathrm{x}_{1}-\mathrm{x}_{1}^{(1)}\right)^{2}+\left(\mathrm{x}_{2}-\mathrm{x}_{2}^{(1)}\right)^{2}<r^{2}\right\} .
$$

Example 2. We consider a peanut-shaped inclusion rotated by an angle $\varphi=5 \pi / 6$ about its centre $\mathbf{x}^{(1)}$

$$
\Omega_{1}=\left\{\mathbf{x} \in \mathbb{R}^{2} \mid \frac{\mathrm{X}_{1}^{2}+\mathrm{X}_{2}^{2}}{a^{2} \cos ^{2} \theta+b^{2} \sin ^{2} \theta}<r^{2}\right\},
$$


where $\theta \in[0,2 \pi)$ is the polar angle of point $\mathbf{x}$ with respect to the centre of the inclusion, $a=1.0$, $b=0.5, r=0.25$, and $\mathrm{X}_{1}$ and $\mathrm{X}_{2}$ are given by

$$
\mathrm{X}_{1}=\left(\mathrm{x}_{1}-\mathrm{x}_{1}^{(1)}\right) \cos \varphi+\left(\mathrm{x}_{2}-\mathrm{x}_{2}^{(1)}\right) \sin \varphi, \quad \mathrm{X}_{2}=-\left(\mathrm{x}_{1}-\mathrm{x}_{1}^{(1)}\right) \sin \varphi+\left(\mathrm{x}_{2}-\mathrm{x}_{2}^{(1)}\right) \cos \varphi .
$$

Example 3. We consider an acorn shaped inclusion centered at $\mathbf{x}^{(1)}$

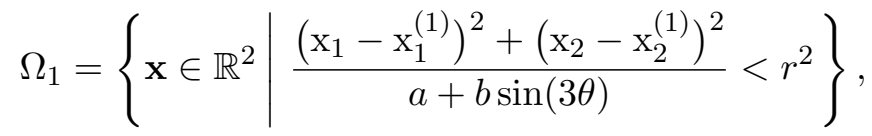

where $\theta \in[0,2 \pi)$ is the polar angle of point $\mathbf{x}$ with respect to the centre of the inclusion, $a=4.25$, $b=2.0$ and $r=0.1$.

Example 4. We consider two inclusions, i.e. $J=2$, namely a circular inclusion of radius $r_{1}=0.25$ and centered at $\mathbf{x}^{(1)}$

$$
\Omega_{1}=\left\{\mathrm{x} \in \mathbb{R}^{2} \mid\left(\mathrm{x}_{1}-\mathrm{x}_{1}^{(1)}\right)^{2}+\left(\mathrm{x}_{2}-\mathrm{x}_{2}^{(1)}\right)^{2}<r_{1}^{2}\right\},
$$

and an elliptical inclusion of semi-axes $a=1.0$ and $b=0.5$, rotated by an angle $\varphi=\pi / 4$ about its centre $\mathbf{x}^{(2)}$

$$
\Omega_{2}=\left\{\mathrm{x} \in \mathbb{R}^{2} \mid\left(\frac{\mathrm{X}_{1}}{a}\right)^{2}+\left(\frac{\mathrm{X}_{2}}{b}\right)^{2}<r_{2}^{2}\right\}
$$

where $r_{2}=0.25$, whilst $\mathrm{X}_{1}$ and $\mathrm{X}_{2}$ are given by equation (16b).

In the present analysis, in the Neumann boundary conditions (6c) and (6d) the amplitude of the applied current was chosen as

$$
I_{0}(\mathbf{x})=-\sin (\theta(\mathbf{x})+\pi / 4)
$$

while its frequency $\omega / 2 \pi$ was set to $500 \mathrm{kHz}$, and we only considered three different values for the time variable, namely $t=0 \mathrm{~s}, t=0.2 \times 10^{-6} \mathrm{~s}$ and $t=0.5 \times 10^{-6} \mathrm{~s}$, respectively.

Generally speaking, the collocation points, $\left\{\mathbf{x}^{(j ; n)}\right\}_{n=1}^{\mathrm{M}_{j}}$, are uniformly distributed on each boundary $\Gamma_{j}, j=\overline{0, J}$. The singularities/sources $\left\{\xi^{(0 ; n)}\right\}_{n=1}^{\mathrm{N}_{0}}, \mathrm{~N}_{0}=\mathrm{N}_{00}+\mathrm{N}_{01}+\ldots+\mathrm{N}_{0 J}$, associated with $\partial \Omega_{0}=\bigcup_{j=0}^{J} \Gamma_{j}$, and $\left\{\xi^{(j ; n)}\right\}_{n=1}^{\mathrm{N}_{j}}$ associated with $\Gamma_{j}, j=\overline{1, J}$ are also uniformly distributed. Moreover, we take $\mathrm{M}_{0}=\mathrm{N}_{00}$ and $\mathrm{M}_{j}=\mathrm{N}_{j}=\mathrm{N}_{0 j}, j=\overline{1, J}$. The aforementioned singularities/sources are preassigned and kept fixed throughout the solution process (i.e. the so-called static MFS approach is employed) on a pseudo-boundary $\widetilde{\Gamma}_{j}$ of a similar shape to that of $\Gamma_{j}$, such that $\operatorname{dist}\left(\widetilde{\Gamma}_{j}, \Gamma_{j}\right), j \in \overline{0, J}$, are fixed constants, see e.g. [10]. Note that, for the examples considered in this paper, the number of inclusions is either $J=1$ (Examples 1-3) or $J=2$ (Example 4).

To illustrate the positioning of the pseudo-boundaries used in the present approach, we consider Example 1 and define $\partial \widetilde{\Omega}_{0}=\widetilde{\Gamma}_{0} \cup \widetilde{\Gamma}_{01}$ and $\widetilde{\Gamma}_{1}$ as follows:

$$
\begin{aligned}
& \widetilde{\Gamma}_{0}=\left\{\mathrm{x} \in \mathbb{R}^{2} \mid \mathrm{x}_{1}^{2}+\mathrm{x}_{2}^{2}=\left[\left(1+d_{0}\right) R\right]^{2}\right\}, \\
& \widetilde{\Gamma}_{01}=\left\{\mathrm{x} \in \mathbb{R}^{2} \mid\left(\mathrm{x}_{1}-\mathrm{x}_{1}^{(1)}\right)^{2}+\left(\mathrm{x}_{2}-\mathrm{x}_{2}^{(1)}\right)^{2}=\left[\left(1-d_{1}\right) r\right]^{2}\right\},
\end{aligned}
$$


and

$$
\widetilde{\Gamma}_{1}=\left\{\mathrm{x} \in \mathbb{R}^{2} \mid\left(\mathrm{x}_{1}-\mathrm{x}_{1}^{(1)}\right)^{2}+\left(\mathrm{x}_{2}-\mathrm{x}_{2}^{(1)}\right)^{2}=\left[\left(1+d_{2}\right) r\right]^{2}\right\}
$$

where $d_{0}>0$ and $d_{1}=d_{2} \in(0,1)$ since $\widetilde{\Gamma}_{01} \subset \Omega_{1}$. Similar positioning of the pseudo-boundaries $\widetilde{\Gamma}_{j}$, $j=\overline{0, J}$, are used in the case where there are $J$ inclusions present (i.e. $d_{0}>0$ is constant associated with the outer boundary $\Gamma_{0}$, and $d_{2 j-1}=d_{2 j}$ associated with the interfaces $\Gamma_{j}, j=\overline{1, J}$ ).

In order to assess the accuracy and convergence of the proposed MFS, for any real-valued function $f: \Gamma_{j} \longrightarrow \mathbb{R}, j=\overline{0, J}$, and any set of points $\left\{\mathbf{x}^{(j ; m)}\right\}_{m=1}^{\mathrm{M}_{j}} \subset \Gamma_{j}$, we define the corresponding root mean square (RMS) error of $f$ on $\Gamma_{j}$ by

$$
\operatorname{err}_{\Gamma_{j}}(f)=\sqrt{\frac{1}{\mathrm{M}_{j}} \sum_{m=1}^{\mathrm{M}_{j}}\left|f\left(\mathbf{x}^{(j ; m)}\right)\right|^{2}}, \quad j=\overline{0, J} .
$$

We also investigate the influence of the number of source points $\mathrm{N}$ and of the distances from the boundaries of the solution domain, $\Gamma_{j}$ to the pseudo-boundaries on which the sources are located, $\widetilde{\Gamma}_{j}$, $j=\overline{0, J}$, on the accuracy. Therefore, we analyse the dependence of the RMS error defined by equation (21) for the real and imaginary Dirichlet (potential) and Neumann (current) transmission conditions (6g) $-(6 \mathrm{j})$ on the interfaces $\Gamma_{j}, j=\overline{1, J}$, with respect to the number of source points and the constants $d_{0}$ and $d_{2 j-1}=d_{2 j}, j=\overline{1, J}$, related to $\operatorname{dist}\left(\widetilde{\Gamma}_{j}, \Gamma_{j}\right), j \in \overline{0, J}$.

We first consider the problem in Example 1 and set $t=0 \mathrm{~s}$ in equation (19), the number of collocation points $\mathrm{M} \in\{80,120,160\}$ and distances $d_{0}=2.0$ and $d_{1}=d_{2}=0.3$, at the same time varying the number of singularities $\mathrm{N}_{0 j} \in\left\{1,2, \ldots, \mathrm{M}_{0 j}\right\}, j=0,1$, i.e. such that $\mathrm{N} \leq \mathrm{M}+2$. Figures $2(\mathrm{a})-(\mathrm{c})$ present the RMS error $\operatorname{err}_{\Gamma_{1}}\left(v_{0}-v_{1}\right)$, which actually measures the accuracy of the potential transmission condition on the interface $\Gamma_{1}$, as a function of the numbers of sources associated with the boundaries $\Gamma_{0}$ and $\Gamma_{1}$ (i.e. $\mathrm{N}_{00}$ and $\mathrm{N}_{01}=\mathrm{N}_{1}$ ), for $\mathrm{M}=80, \mathrm{M}=120$ and $\mathrm{M}=160$, respectively. For all values of the number $\mathrm{M}$ of boundary collocation points considered, the following conclusions can be drawn from these figures:

(i) For a fixed value of the number $\mathrm{N}_{00} \in\left\{1,2, \ldots, \mathrm{M}_{00}\right\}$ of source points corresponding to the outer boundary $\Gamma_{0}$, the RMS error $\operatorname{err}_{\Gamma_{1}}\left(v_{0}-v_{1}\right)$ decreases as the number of singularities $\mathrm{N}_{01}$ associated with the interface $\Gamma_{1}$ increases.

(ii) Although not illustrated in Figures 2(a)-(c), it should be mentioned that, for fixed but large admissible values of $d_{1}=d_{2}$ (i.e. $d_{1}=d_{2}$ approaching 1.0) and a fixed value $\mathrm{N}_{00} \in\left\{1,2, \ldots, \mathrm{M}_{00}\right\}$, there exists a threshold value of the parameter $\mathrm{N}_{01}$, say $\widetilde{\mathrm{N}}_{01}$, after which $\operatorname{err}_{\Gamma_{1}}\left(v_{0}-v_{1}\right)$ starts to increase as $\mathrm{N}_{01} \longrightarrow \mathrm{M}_{01}$, the distribution of this RMS error becomes irregular and, consequently, the MFS approximations for the Dirichlet transmission condition on the interface $\Gamma_{1}$ are more inaccurate. A possible reason for this increment in the inaccuracies of the MFS approximations for the real part of the potential transmission conditions on the interface $\Gamma_{1}$ is the fact that increasing the number $\mathrm{N}_{00}$ of sources corresponding to $\Gamma_{1}$ results in a highly ill-conditioned matrix $\mathbf{A}$ for the problem in Example 1.

(iii) For a fixed value of the number $\mathrm{N}_{01} \in\left\{1,2, \ldots, \widetilde{\mathrm{N}}_{01}\right\}$ of source points corresponding to the interface $\Gamma_{1}$, the error in the Dirichlet transmission condition on $\Gamma_{1}, \operatorname{err}_{\Gamma_{1}}\left(v_{0}-v_{1}\right)$, has almost the same value for all $\mathrm{N}_{00} \geq 5$.

(iv) For all $\mathrm{N}_{00} \geq 5$ a fixed value of the number of source points corresponding to the interface $\Gamma_{1}$ which is set to be proportional to the maximum admissible number of source points on $\Gamma_{1}$, e.g. $\mathrm{N}_{01}=\mathrm{M}_{01} / 2$, the RMS error $\operatorname{err}_{\Gamma_{1}}\left(v_{0}-v_{1}\right)$ decreases with respect to the number of boundary collocation points, M, i.e. the MFS is convergent with respect to increasing M. 
The same conclusions can be drawn from Figures 3(a)-(c) for the RMS error corresponding to the transmission condition for the real part of the electric current on the interface $\Gamma_{1}$, $\operatorname{err}_{\Gamma_{1}}\left[\mathbf{n}^{(0)} \cdot\left(\sigma_{0} \nabla v_{0}-\right.\right.$ $\left.\left.\omega \varepsilon_{0} \nabla h_{0}\right)+\mathbf{n}^{(1)} \cdot\left(\sigma_{1} \nabla v_{1}-\omega \varepsilon_{1} \nabla h_{1}\right)\right]$. The transmission conditions on $\Gamma_{1}$ associated with the imaginary parts of the potential and current display similar behaviours, although these have not been presented here. As expected, $\operatorname{err}_{\Gamma_{1}}\left(v_{0}-v_{1}\right)<\operatorname{err}_{\Gamma_{1}}\left[\mathbf{n}^{(0)} \cdot\left(\sigma_{0} \nabla v_{0}-\omega \varepsilon_{0} \nabla h_{0}\right)+\mathbf{n}^{(1)} \cdot\left(\sigma_{1} \nabla v_{1}-\omega \varepsilon_{1} \nabla h_{1}\right)\right]$ for the fixed values of the MFS parameters considered, namely $t=0 \mathrm{~s}$ in equation (19), the number of collocation points $\mathrm{M} \in\{80,120,160\}$ and distances $d_{0}=2.0$ and $d_{1}=d_{2}=0.3$, and all admissible values of the numbers of source points $\mathrm{N}_{00} \in\left\{5,6, \ldots, \mathrm{M}_{00}\right\}$ and $\mathrm{N}_{01} \in\left\{1,2, \ldots, \widetilde{\mathrm{N}}_{01}\right\}$, i.e. the MFS approximations for the Neumann (current) transmission conditions on the interface $\Gamma_{1}$ are more inaccurate than the numerical results retrieved for the Dirichlet (potential) transmission conditions on $\Gamma_{1}$.

It is well-known that the accuracy of the MFS depends on the distance between the pseudo-boundary on which the singularities are located and the boundary of the solution domain. For direct problems with exact boundary data, it is generally advised to place the singularities as far away from the boundary of the domain under investigation as possible, as much as the machine precision allows. Ramachandran [37] showed that the singular value decomposition (SVD) could mitigate this critical dependence.

However, the direct problem investigated in this paper is somehow different from the situation presented above. More specifically, in the case of direct problems in EIT, the domain occupied by the background $\Omega_{0}$ is a multiply connected domain and, therefore, there exist source points associated with the interfaces $\Gamma_{j}, j=\overline{1, J}$, and corresponding to the background which are located inside the simply connected domain $\Omega$, more precisely, these sources are situated in the domains occupied by the inclusions $\Omega_{j} \subset \Omega, j=\overline{1, J}$. Consequently, the distances between the interfaces $\Gamma_{j}$ and their corresponding pseudoboundaries on which the MFS sources are located, and hence $d_{2 j-1}=d_{2 j}, j=\overline{1, J}$, are constrained by the size of the inclusions $\Omega_{j} \subset \Omega_{0}, j=\overline{1, J}$. Nonetheless, it should be mentioned that there is no constraint on the distance between the outer boundary $\Gamma_{0}$ and its associated pseudo-boundary $\widetilde{\Gamma}_{0}$ and this can have any positive value.

Figures 4(a)-(c) present the RMS error $\operatorname{err}_{\Gamma_{1}}\left(h_{0}-h_{1}\right)$ as a function of the distances $d_{0} \in[0.05,3.0]$ and $d_{1}=d_{2} \in[0.01,0.9]$ from the boundary $\Gamma_{j}$ to the pseudo-boundary $\widetilde{\Gamma}_{j}, j=0,1$, on which the sources are positioned, obtained using $t=0.5 \times 10^{-6} \mathrm{~s}$ and various numbers of collocation and source points, namely $\mathrm{M}=\mathrm{N}=80, \mathrm{M}=\mathrm{N}=120$ and $\mathrm{M}=\mathrm{N}=160$, respectively, for Example 1. It can be observed from these figures that, as the numbers of boundary collocation points $\mathrm{M}$ and singularities $\mathrm{N}$ increase, $d_{0}$ increases and $d_{1}=d_{2} \longrightarrow 0.90$, the distribution of the RMS error $\operatorname{err}_{\Gamma_{1}}\left(h_{0}-h_{1}\right)$ becomes irregular. This is a direct consequence of the fact that the corresponding MFS matrix A becomes highly ill-conditioned since not only the dimension of this matrix increases (i.e. $\mathrm{M}=\mathrm{N}$ increase), but also the singularities on the pseudo-boundary $\widetilde{\Gamma}_{1}$ associated with the interface $\Gamma_{1}$ cluster around the origin. Similar results have been obtained for the RMS error defined by equation (21) and corresponding to the imaginary part of the Neumann (current) transmission condition on $\Gamma_{1}$ and these are displayed in Figures 5(a)-(c). Although not illustrated, similar results were also obtained, in the case of Example 1, for the RMS errors $\operatorname{err}_{\Gamma_{1}}\left(v_{0}-v_{1}\right)$ and $\operatorname{err}_{\Gamma_{1}}\left[\mathbf{n}^{(0)} \cdot\left(\sigma_{0} \nabla v_{0}-\omega \varepsilon_{0} \nabla h_{0}\right)+\mathbf{n}^{(1)} \cdot\left(\sigma_{1} \nabla v_{1}-\omega \varepsilon_{1} \nabla h_{1}\right)\right]$, as well as the other examples analysed in this study. According to the RMS errors associated with the transmission conditions presented in Figures 4 and 5, for stability and accuracy reasons it is recommended to choose the source points corresponding to the interfaces (inner boundaries) $\Gamma_{j}, j=\overline{1, J}$, on the pseudoboundaries $\widetilde{\Gamma}_{j}, j=\overline{1, J}$, such that $d_{2 j-1}=d_{2 j} \in[0.02,0.35], j=\overline{1, J}$.

The numerical results presented in the sequel have been obtained by setting the corresponding MFS parameters introduced in Section 3 as follows:

(i) $\mathrm{M}_{00} \in\{40,60,80\}$ and $\mathrm{M}_{0 j}=\mathrm{M}_{j} \in\{20,30,40\}$ for $j=1,2$, such that $\mathrm{M} \in\{80,120,160\}$ for Examples $1-3$ and $\mathrm{M} \in\{120,180,240\}$ for Example 4;

(ii) $\mathrm{N}_{0 j}=\mathrm{M}_{0 j}$ for $j=0,1,2$, such that $\mathrm{M}=\mathrm{N}$ for Examples 1-4; 
(iii) $d_{0}=2.0$ and $d_{1}=d_{2}=0.3$ (Example 1$) ; d_{0}=1.0$ and $d_{1}=d_{2}=0.2$ (Example 2); $d_{0}=2.0$ and $d_{1}=d_{2}=0.2$ (Example 3$) ; d_{0}=1.0, d_{1}=d_{2}=0.3$ and $d_{3}=d_{4}=0.3$ (Example 4 ).

For Examples 1-4, $d_{1}$ and $d_{2}$ set the positions of the pseudo-boundaries corresponding to $\Gamma_{1}$, whilst the distances between $\Gamma_{2}$ and its corresponding pseudo-boundaries in Example 4 are determined by $d_{3}$ and $d_{4}$.

Figures 6 and 7 present the contour plots of the real and imaginary parts of the electric potential $\phi$, $v$ and $h$, respectively, obtained in the domain corresponding to Example 1 by applying the boundary current given by equation (19) with $\mathrm{M}=\mathrm{N}=160$ and various values of the time variable, namely $t=0 \mathrm{~s}, t=0.2 \times 10^{-6} \mathrm{~s}$ and $t=0.5 \times 10^{-6} \mathrm{~s}$. It can be seen from Figures $6(\mathrm{a})$ and (b) that although the numerically reconstructed values of $v=\operatorname{Re} \phi$ at $t=0 \mathrm{~s}$ and $t=0.2 \times 10^{-6} \mathrm{~s}$ are different, the distributions of the real electric potential in $\Omega$ are very similar. However, the real electric potential numerically retrieved at $t=0.5 \times 10^{-6} \mathrm{~s}$ and illustrated in Figure 6(c) clearly accounts for the presence of the circular inclusion $\Omega_{1}$ given by equation (15).

As expected, the behaviour of the reconstructed imaginary part of the electric potential $h=\operatorname{Im} \phi$ is opposite to that of the real electric potential $v=\operatorname{Re} \phi$, see Figures 6 and 7 . More precisely, at $t=0 \mathrm{~s}$ and $t=0.2 \times 10^{-6} \mathrm{~s}$, although their numerical values are different, both imaginary electric potential distributions account for the presence of the circular inclusion $\Omega_{1}$, see Figures 7 (a) and (b). On the contrary, at $t=0.5 \times 10^{-6} \mathrm{~s}$ the presence of the inclusion $\Omega_{1}$ has little effect on the numerically reconstructed values of the imaginary part of the electric potential $h=\operatorname{Im} \phi$ presented in Figure 7 (c).

The approach developed here was also shown to be efficient, accurate and convergent with respect to increasing the number of boundary collocation points when the inclusion $\Omega_{1}$ was assumed to be smooth, but non-convex. Two examples have been considered to illustrate this situation, namely the peanut-shaped inclusion considered in Example 2 for $t=0.2 \times 10^{-6} \mathrm{~s}$ and the acorn-shaped inclusion in Example 3 for $t=0.5 \times 10^{-6} \mathrm{~s}$. The contour plots of the numerically retrieved values for $v$ and $h$ in $\Omega$ and corresponding to Examples 2 and 3 are illustrated in Figures $8-11$.

The proposed procedure works equally well when two inclusions $(J=2)$ are present in the background medium as is the case in Example 4, in which we consider a circular inclusion and an elliptical one given by equations (18a) and (18b), respectively. Figures 12 and 13 present the contour plots of the retrieved real, $v$, and imaginary parts of the potential, $h$, respectively, using the boundary current given by equation (19) at $t=0.5 \times 10^{-6} \mathrm{~s}$ and various numbers of collocation and source points, i.e. $\mathrm{M}=\mathrm{N} \in\{80,120,160\}$. Overall, from Figures $6-13$ we can conclude that the MFS provides accurate and convergent numerical solutions for the real and imaginary parts of the electric potential in the background medium $\Omega_{0}$ as well as in the inclusions $\Omega_{j} \subset \Omega, j=\overline{1, J}$, at all time instants $t \geq 0$.

\section{CONCLUSions}

In this paper, the MFS has been implemented and analysed for obtaining the numerical solution of the complex EIT forward problem in two dimensions. A generalized MFS formulation for this EIT direct problem has been developed for the case when a finite number of inclusions with constant physical properties (i.e. conductivity and permittivity) are assumed to be compactly contained in a given medium (background). The efficiency, accuracy and numerical convergence of the proposed method have been investigated and validated by considering five numerical examples corresponding to various smooth convex or non-convex shapes of the inclusion(s), namely circular, elliptic, peanut-shaped and acorn-shaped.

The proposed technique is efficient and can be extended to other problems for complex EIT: threedimensional forward problems, inverse geometric problems (i.e. reconstruction of the inclusion(s) in the background when the physical properties of both the inclusion(s) and the background are known 
a priori), parameter identification problems (i.e. identification of the physical properties of the inclusion(s) in the background) and combined geometric-parameter identification problems in two and three dimensions. However, these extensions will be considered in future work.

Acknowledgements. L. Marin acknowledges the financial support received from the Romanian National Authority for Scientific Research (CNCS-UEFISCDI), project number PN-II-ID-PCE-2011-30521. The authors also thank the reviewers for their constructive comments and suggestions, which significantly contributed to improving the quality of the publication.

\section{References}

[1] Borcea L. Electrical impedance tomography. Inverse Problems 2002; 18:R99-R136.

[2] Cheney M, Isaacson D, Newell JC, Simske S, Goble J. NOSER: an algorithm for solving the inverse conductivity problem. International Journal for Imaging Systems and Technology 1990; 2:66-75.

[3] Berger JA, Karageorghis A. The method of fundamental solutions for heat conduction in layered materials. International Journal for Numerical Methods in Engineering 1999; 45:1681-1694.

[4] Berger JA, Karageorghis A. The method of fundamental solutions for layered elastic materials. Engineering Analysis with Boundary Elements 2001; 25:877-886.

[5] Fairweather G, Karageorghis A. The method of fundamental solutions for elliptic boundary value problems. Advances in Computational Mathematics 1998; 9:69-95.

[6] Fairweather G, Karageorghis A, Martin PA. The method of fundamental solutions for scattering and radiation problems. Engineering Analysis with Boundary Elements 2003; 27:759-769.

[7] Folland GB. Introduction to Partial Differential Equations. Princeton University Press. Princeton, NJ, 1995.

[8] Gabriel S, Lau RW, Gabriel C. The dielectric properties of biological tissues: II. Measurements in the frequency range $10 \mathrm{~Hz}$ to 20GHz. Physics in Medicine and Biology 1996; 41:2251-2269.

[9] Golberg MA, Chen CS. The method of fundamental solutions for potential, Helmholtz and diffusion problems. In: Golberg MA (Ed.), Boundary Integral Methods: Numerical and Mathematical Aspects. Computational Engineering, vol. 1, WIT Press/Computational Mechanics Publications, Boston, MA, 1999, pp. 103-176.

[10] Gorzelańczyk P, Kołodziej JA. Some remarks concerning the shape of the shape contour with application of the method of fundamental solutions to elastic torsion of prismatic rods. Engineering Analysis with Boundary Elements 2008; 32:64-75.

[11] Harrach B, Seo JK Seo, Woo EJ. Factorization method and its physical justification in frequencydifference electrical impedance tomography. IEEE Transactions on Medical Imaging 2010; 29:19181926.

[12] Holder D. Electrical Impedance Tomography: Methods, History and Applications. Institute of Physics Publishing. Bristol, UK, 2005.

[13] Jun SC, Kuen J, Lee J, Woo EJ, Holder D, Seo JK. Frequency-diffrenece EIT (fdEIT) using weighted difference and equivalent homogeneous admittivity: validation by simulation and tank experiment. Physiological Measurements 2009; 30:1087-1099. 
[14] Karageorghis A. Modified methods of fundamental solutions for harmonic and biharmonic problems with boundary singularities. Numerical Methods for Partial Differential Equations 1992; 8:1-19.

[15] Karageorghis A. The method of fundamental solutions for the calculation of the eigenvalues of the Helmholtz equation. Applied Mathematics Letters 2001; 14:837-842.

[16] Karageorghis A, Fairweather G. The method of fundamental solutions for the numerical solution of the biharmonic equation. Journal of Computational Physics 1987; 69:434-459.

[17] Karageorghis A, Fairweather G. The method of fundamental solutions for axisymmetric elasticity problems. Computational Mechanics 2000; 25:524-532.

[18] Karageorghis A, Lesnic D. Steady-state nonlinear heat conduction in composite materials using the method of fundamental solutions. Computer Methods in Applied Mechanics and Engineering $2008 ; 197: 3122-3137$.

[19] Karageorghis A, Smyrlis Y-S. Matrix decomposition MFS algorithms for elasticity and thermoelasticity problems in axisymmetric domains. Journal of Computational and Applied Mathematics 2007; 206:774-795.

[20] Karageorghis A, Lesnic D, Marin L. A survey of applications of the MFS to inverse problems. Inverse Problems in Science and Engineering 2011; 19:309-336.

[21] Karageorghis A, Poullikkas A, Berger JR. Stress intensity factor computation using the method of fundamental solutions. Computational Mechanics 2006; 37:445-454.

[22] Kim S, Lee EJ, Woo EJ, Seo JK. Asymptotic analysis of the membrane structure to sensitivity of frequency-difference electrical impedance tomography. Inverse Problems 2012; 28:17pp.

[23] Kupradze VD, Aleksidze MA. The method of functional equations for the approximate solution of certain boundary value problems. USSR Computational Mathematics and Mathematical Physics $1964 ; 4: 82-126$.

[24] Lionheart W, Polydorides N, Borsic A. The reconstruction problem. In: Holder DS (Ed.), Electrical Impedance Tomography: Methods, History and Applications. Institute of Physics Publishing, Bristol, UK, 2005.

[25] Marin L. Stable MFS solution to singular direct and inverse problems associated with the Laplace equation subjected to noisy data. CMES: Computer Modeling in Engineering \& Sciences 2008; $37: 203-242$

[26] Marin L. Treatment of singularities in the method of fundamental solutions for two-dimensional Helmholtz-type equations. Applied Mathematical Modelling 2010; 34:1615-1633.

[27] Marin L. A meshless method for the stable solution of singular inverse problems for two-dimensional Helmholtz-type equations. Engineering Analysis with Boundary Elements 2010; 34:274-288.

[28] Marin L, Karageorghis A. The MFS-MPS for two-dimensional steady-state thermoelasticity problems. Engineering Analysis with Boundary Elements 2013; 37:1004-1020.

[29] Marin L, Lesnic D. The method of fundamental solutions for nonlinear functionally graded materials. International Journal of Solids and Structures 2007; 44:6878-6890.

[30] Mathon R, Johnston RL. The approximate solution of elliptic boundary value problems by fundamental solutions. SIAM Journal on Numerical Analysis 1977; 14:638-650. 
[31] Metherall P, Barber DC, Smallwood RH, Brown BH. Three-dimensional electrical impedance tomography. Nature 1996; 380:509-512.

[32] Oh TI, Koo W, Lee KH, Kim SM, Lee J, Kim SW, Seo JK, Woo EJ. Validation of multifrequency electrical impedance tomography (mfEIT) KHU Mark 1: impedance spectroscopy and time-difference imaging. Physiological Measurements 2008; 28:1175-1188.

[33] Poullikkas A, Karageorghis A, Georgiou G. Methods of fundamental solutions for harmonic and biharmonic boundary value problems. Computational Mechanics 1998; 21:416-423.

[34] Poullikkas A, Karageorghis A, Georgiou G. The method of fundamental solutions for inhomogeneous elliptic problems. Computational Mechanics 1998; 22:100-107.

[35] Poullikkas A, Karageorghis A, Georgiou G. The numerical solution of three-dimensional Signorini problems with the method of fundamental solutions. Engineering Analysis with Boundary Elements 2001; 25:221-227.

[36] Poullikkas A, Karageorghis A, Georgiou G. The numerical solution for three-dimensional elastostatics problems. Computers and Structures 2002; 80:365-370.

[37] Ramachandran PA. Method of fundamental solutions: Singular value decomposition analysis. Communications in Numerical Methods in Engineering 2002; 18:789-801.

[38] Seo JK, Lee J, Kim SW, Zribi H, Woo EJ. Frequency-difference electrical impedance tomography (fdEIT): algorithm development and feasibility study. Physiological Measurements 2008; 29:929944.

[39] Tsai CC. The method of fundamental solutions with dual reciprocity for three-dimensional thermoelasticity under arbitrary forces. International Journal for Computer-Aided Engineering and Software 2009; 26:229-244.

[40] Woo EJ, Hua P, Webster JG, Tompkins WJ. A robust image reconstruction algorithm and its parallel implementation in electrical impedance tomography. IEEE Transactions on Medical Imaging 1993; 12:137-146.

[41] Yorkey TJ, Webster JG, Tompkins WJ. Comparing reconstruction algorithms for electrical impedance tomography. IEEE Transactions on Biomedical Engineering 1987; BME-34:843-852. 


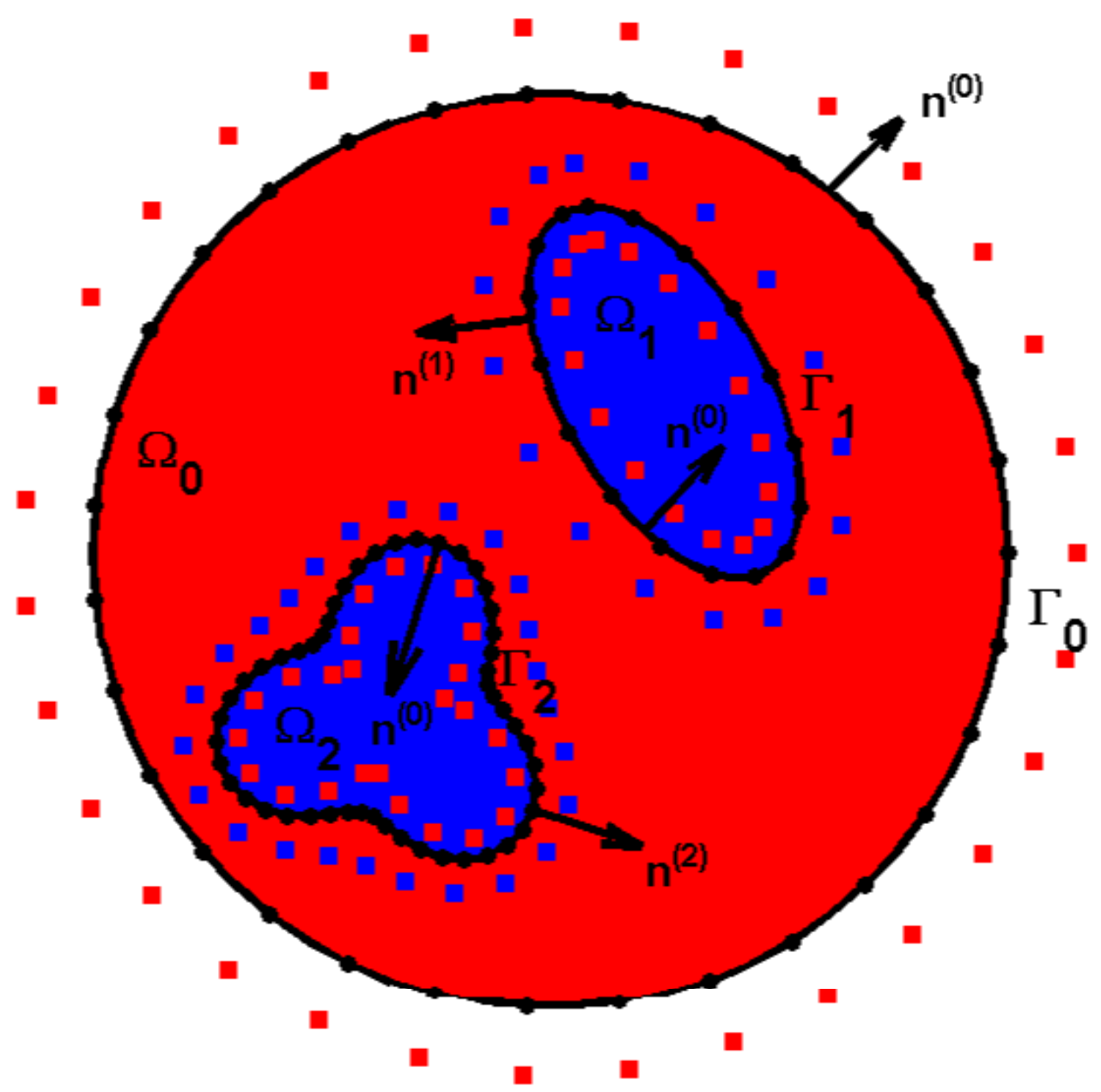

Figure 1: Geometry of the problem. Possible placement of the sources for $\Omega_{0}(\boldsymbol{\square})$ and $\Omega_{j}, j=1,2,(\mathbb{\square})$, and the collocation points $(\bullet)$. 


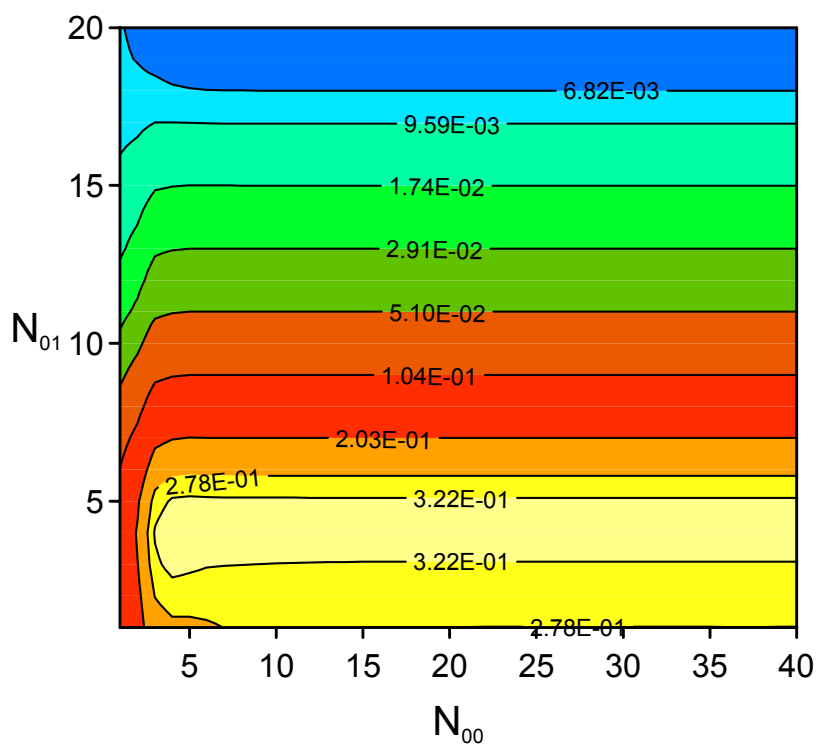

(a) Example 1: $\mathrm{M}=80$

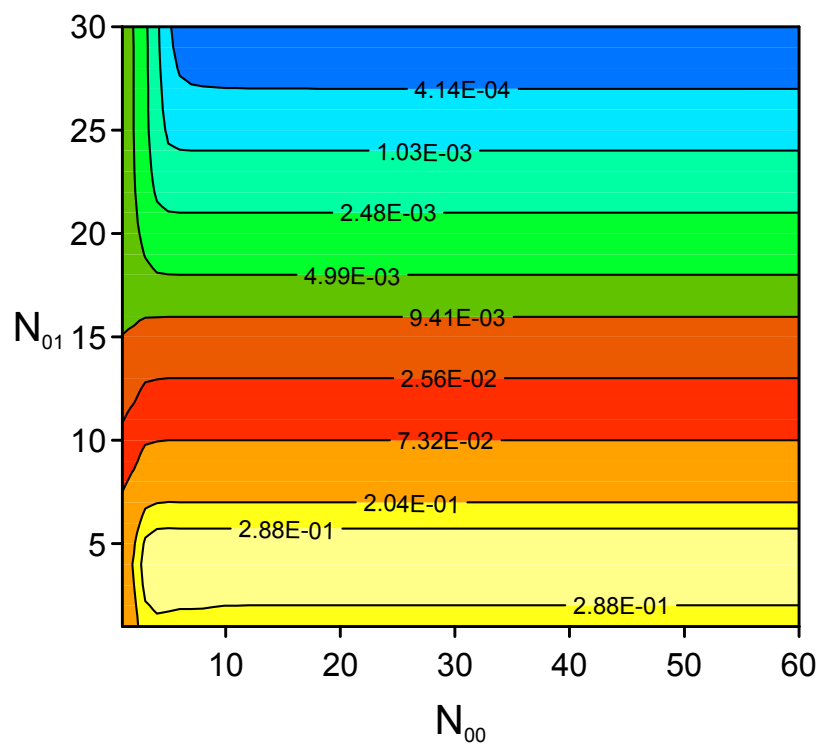

(b) Example 1: $\mathrm{M}=120$

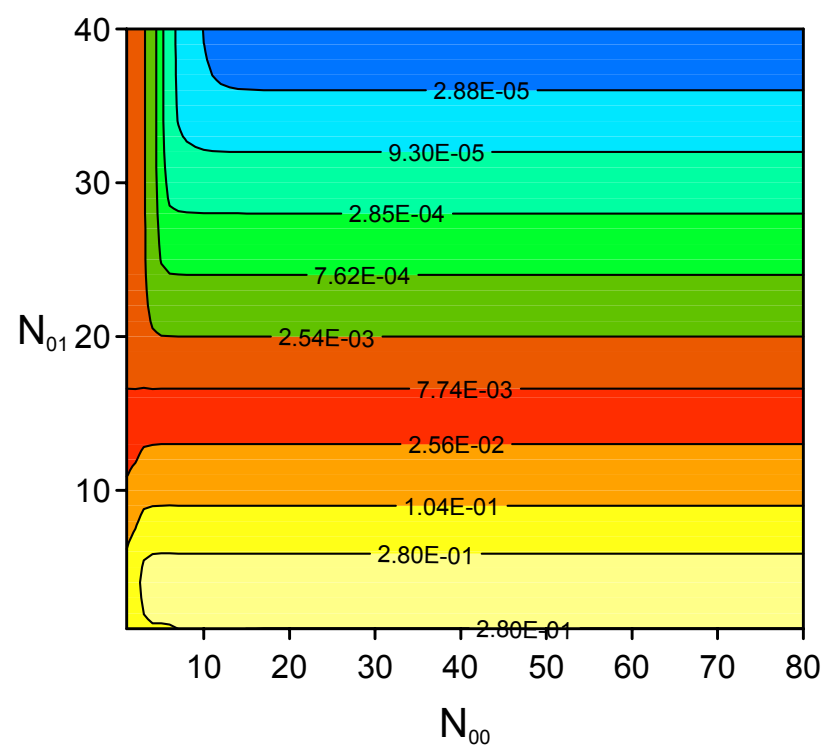

(c) Example 1: $\mathrm{M}=160$

Figure 2: The RMS error, $\operatorname{err}_{\Gamma_{1}}\left(v_{0}-v_{1}\right)$, as a function of the number of sources $\mathrm{N}_{00} \in\left\{1,2, \ldots, \mathrm{M}_{00}\right\}$ and $\mathrm{N}_{01} \in\left\{1,2, \ldots, \mathrm{M}_{01}\right\}$ at $t=0 \mathrm{~s}$ and various numbers of collocation points, namely (a) $\mathrm{M}=80$, (b) $\mathrm{M}=120$ and (c) $\mathrm{M}=160$, for Example 1 . 


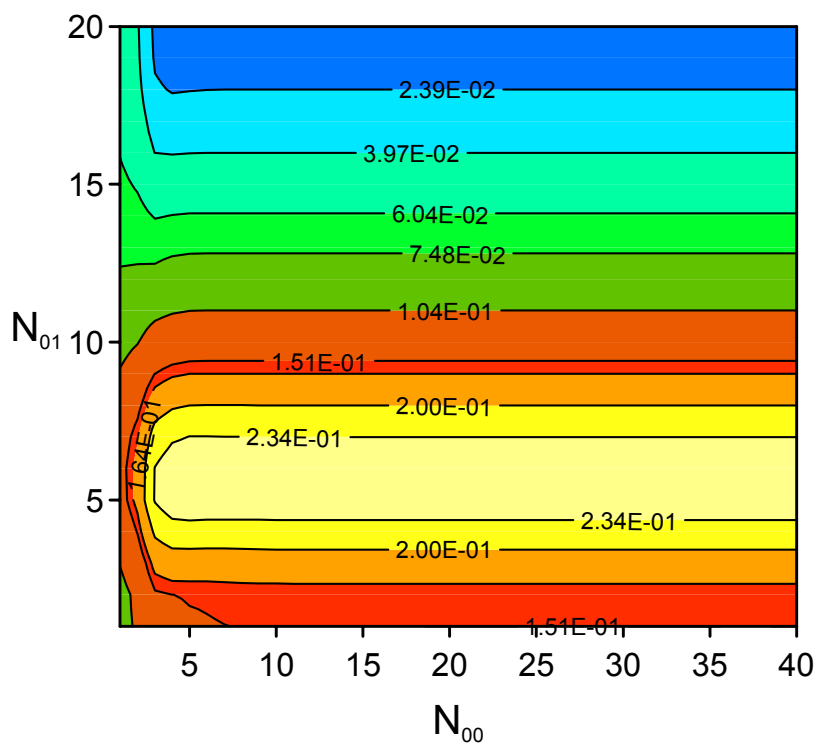

(a) Example 1: $\mathrm{M}=80$

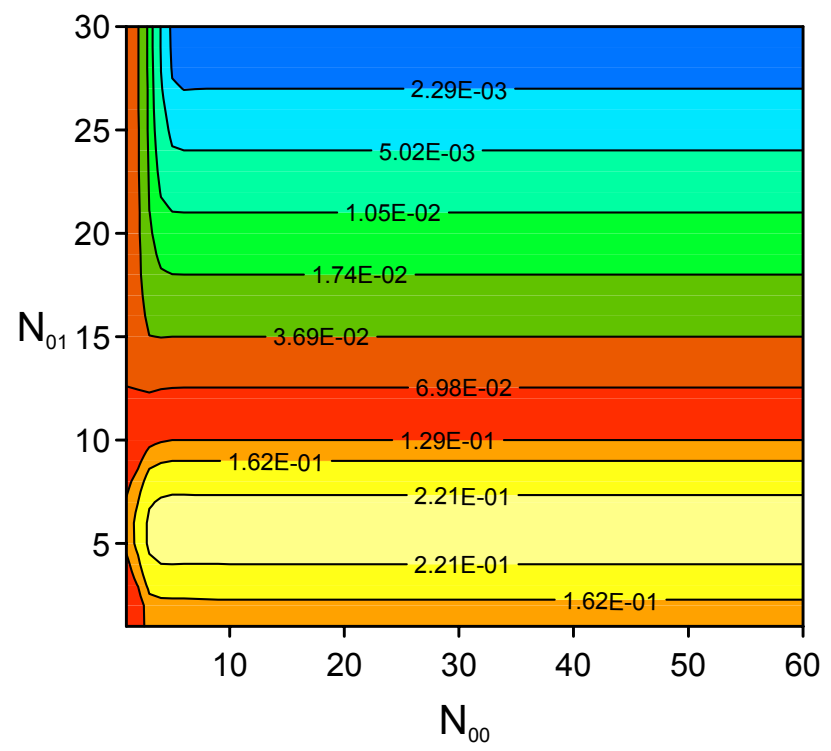

(b) Example 1: $\mathrm{M}=120$

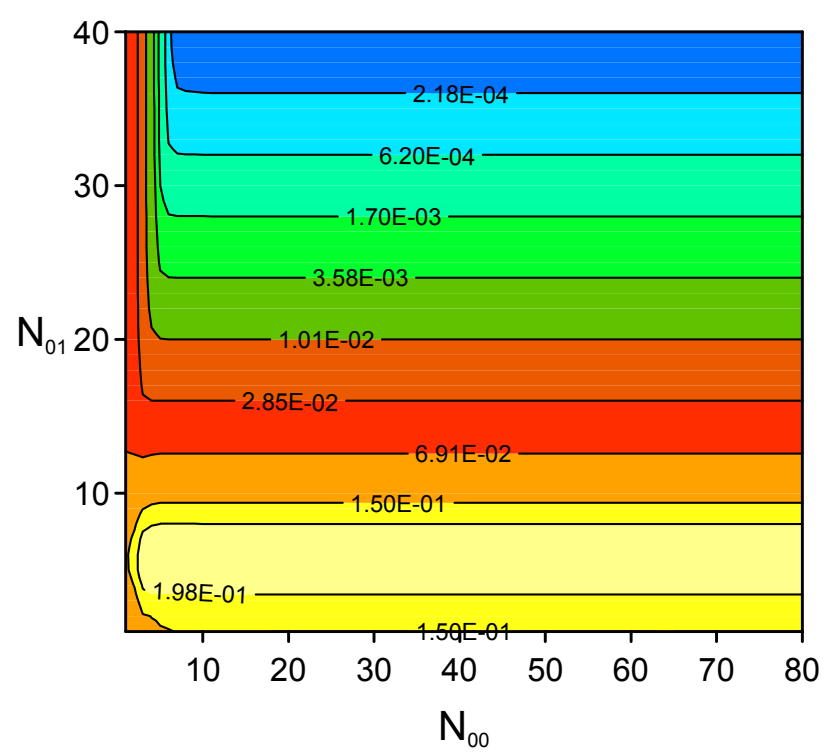

(c) Example 1: $\mathrm{M}=160$

Figure 3: The RMS error, $\operatorname{err}_{\Gamma_{1}}\left[\mathbf{n}^{(0)} \cdot\left(\sigma_{0} \nabla v_{0}-\omega \varepsilon_{0} \nabla h_{0}\right)+\mathbf{n}^{(1)} \cdot\left(\sigma_{1} \nabla v_{1}-\omega \varepsilon_{1} \nabla h_{1}\right)\right]$, as a function of the number of sources $\mathrm{N}_{00} \in\left\{1,2, \ldots, \mathrm{M}_{00}\right\}$ and $\mathrm{N}_{01} \in\left\{1,2, \ldots, \mathrm{M}_{01}\right\}$ at $t=0$ s and various numbers of collocation points, namely (a) $\mathrm{M}=80$, (b) $\mathrm{M}=120$ and (c) $\mathrm{M}=160$, for Example 1 . 


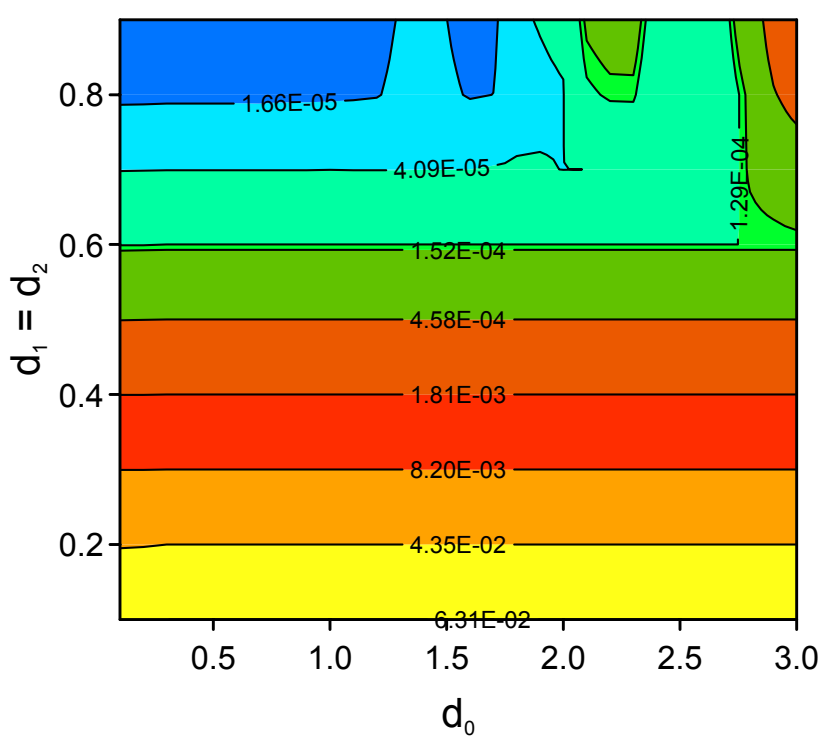

(a) Example 1: $\mathrm{M}=\mathrm{N}=80$

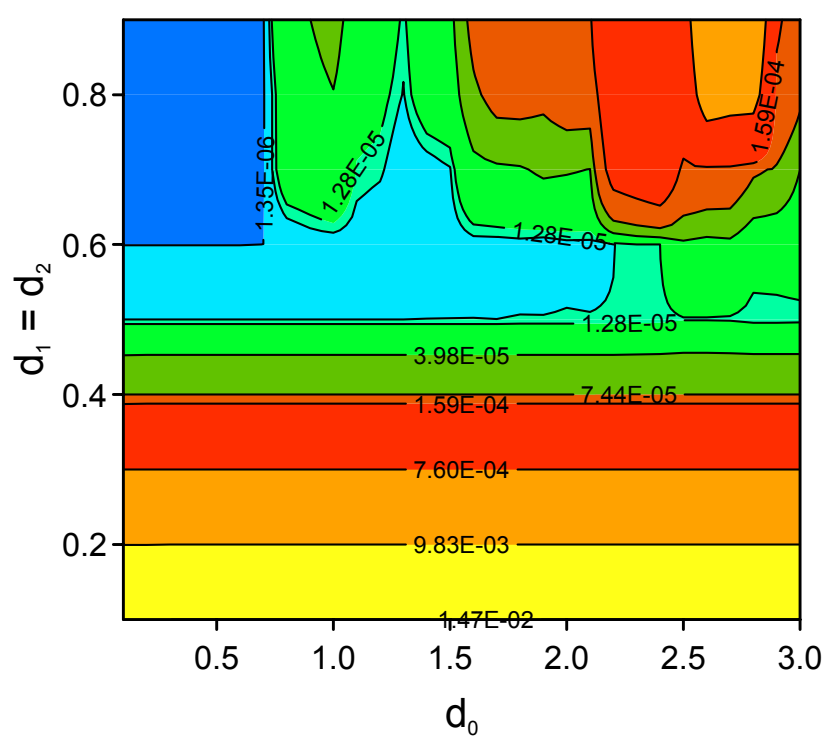

(b) Example 1: $\mathrm{M}=\mathrm{N}=120$

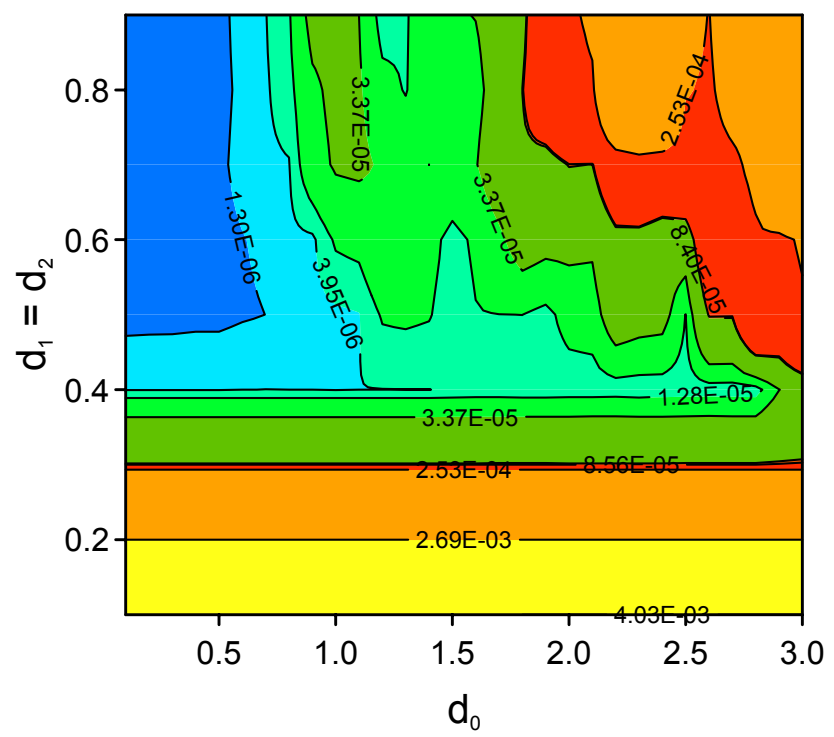

(c) Example 1: $\mathrm{M}=\mathrm{N}=160$

Figure 4: The RMS error, $\operatorname{err}_{\Gamma_{1}}\left(h_{0}-h_{1}\right)$, as a function of $d_{0}$ and $d_{1}=d_{2}$ at $t=0.5 \times 10^{-6} \mathrm{~s}$ and various numbers of collocation and source points, namely (a) $\mathrm{M}=\mathrm{N}=80$, (b) $\mathrm{M}=\mathrm{N}=120$ and (c) $\mathrm{M}=\mathrm{N}=160$, for Example 1 . 


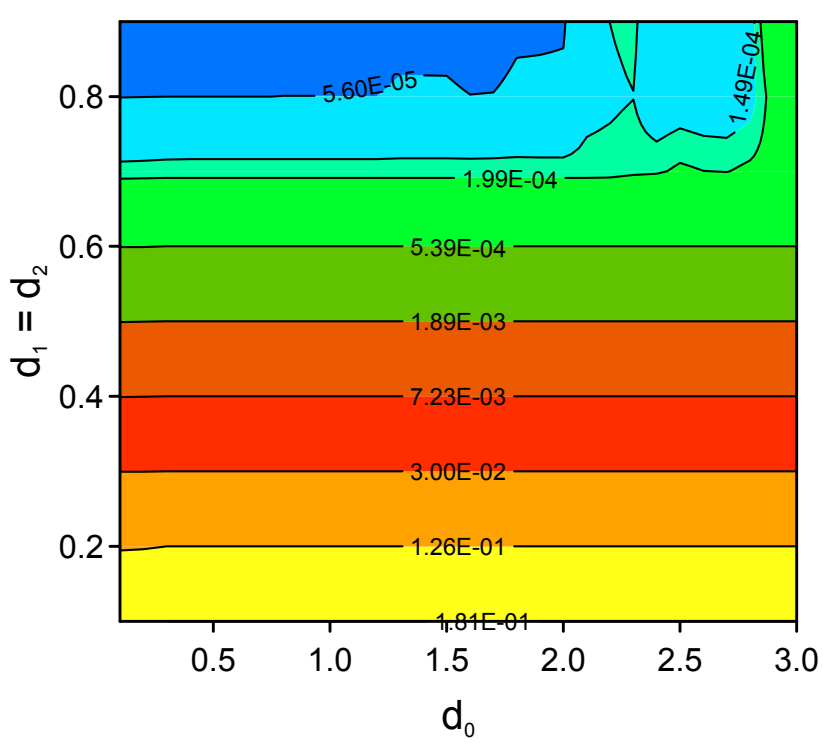

(a) Example 1: $\mathrm{M}=\mathrm{N}=80$

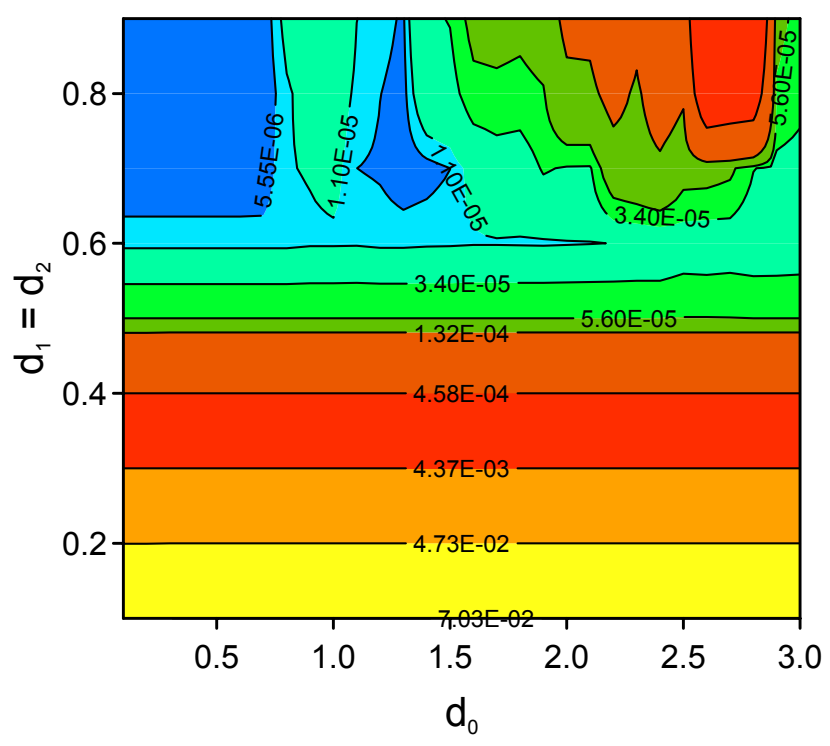

(b) Example 1: $\mathrm{M}=\mathrm{N}=120$

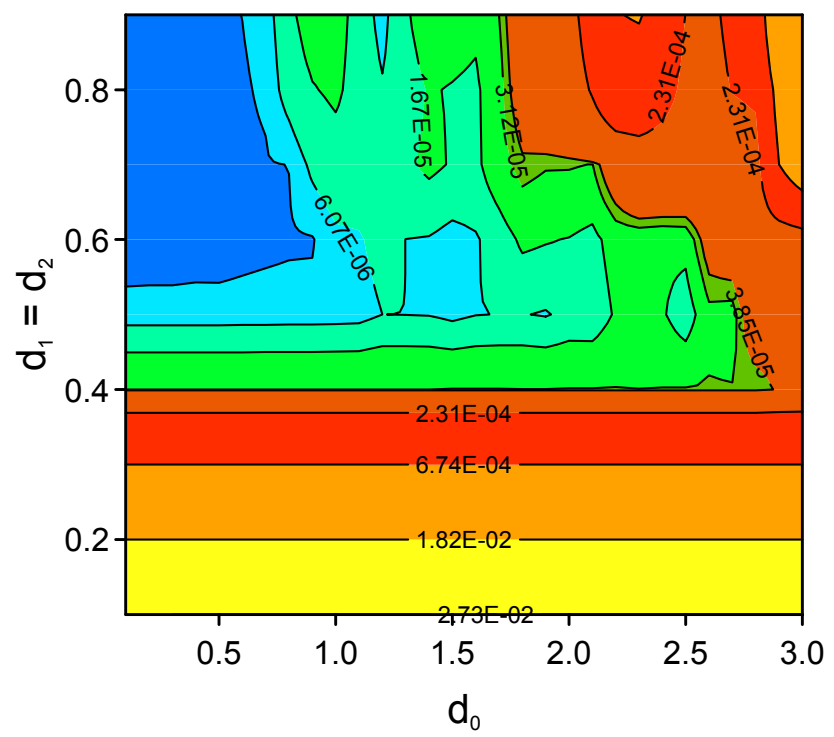

(c) Example 1: $\mathrm{M}=\mathrm{N}=160$

Figure 5: The RMS error, $\operatorname{err}_{\Gamma_{1}}\left[\mathbf{n}^{(0)} \cdot\left(\omega \varepsilon_{0} \nabla v_{0}+\sigma_{0} \nabla h_{0}\right)+\mathbf{n}^{(1)} \cdot\left(\omega \varepsilon_{1} \nabla v_{1}+\sigma_{1} \nabla h_{1}\right)\right]$, as a function of the distances $d_{0}$ and $d_{1}=d_{2}$ at $t=0.5 \times 10^{-6} \mathrm{~s}$ and various numbers of collocation and source points, namely (a) $\mathrm{M}=\mathrm{N}=80$, (b) $\mathrm{M}=\mathrm{N}=120$ and (c) $\mathrm{M}=\mathrm{N}=160$, for Example 1 . 


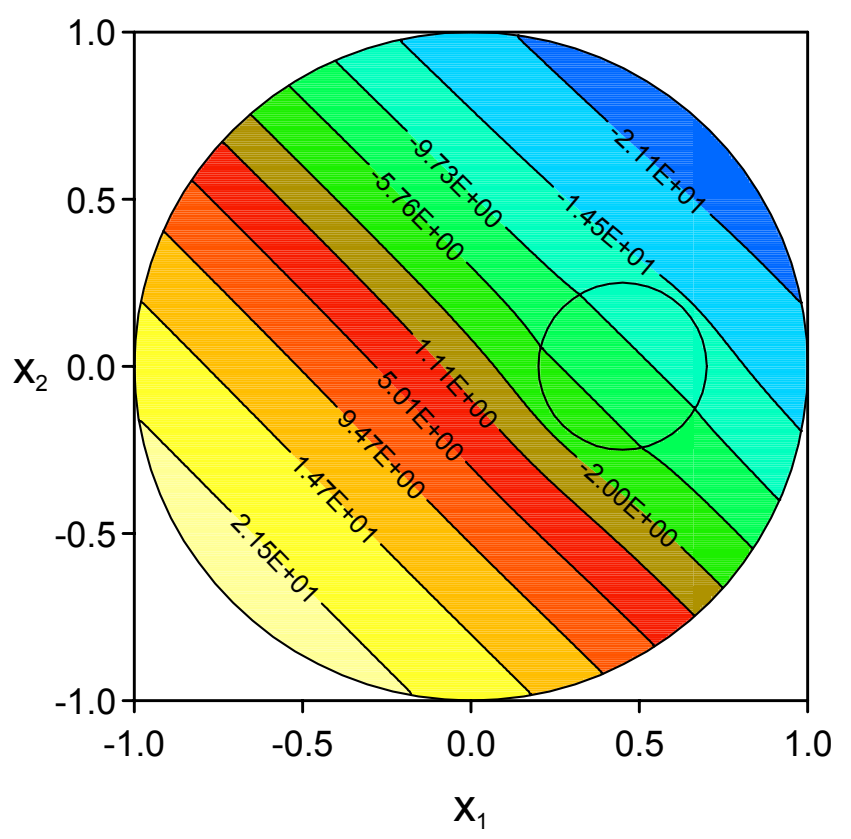

(a) Example 1: $v(t=0 \mathrm{~s})$

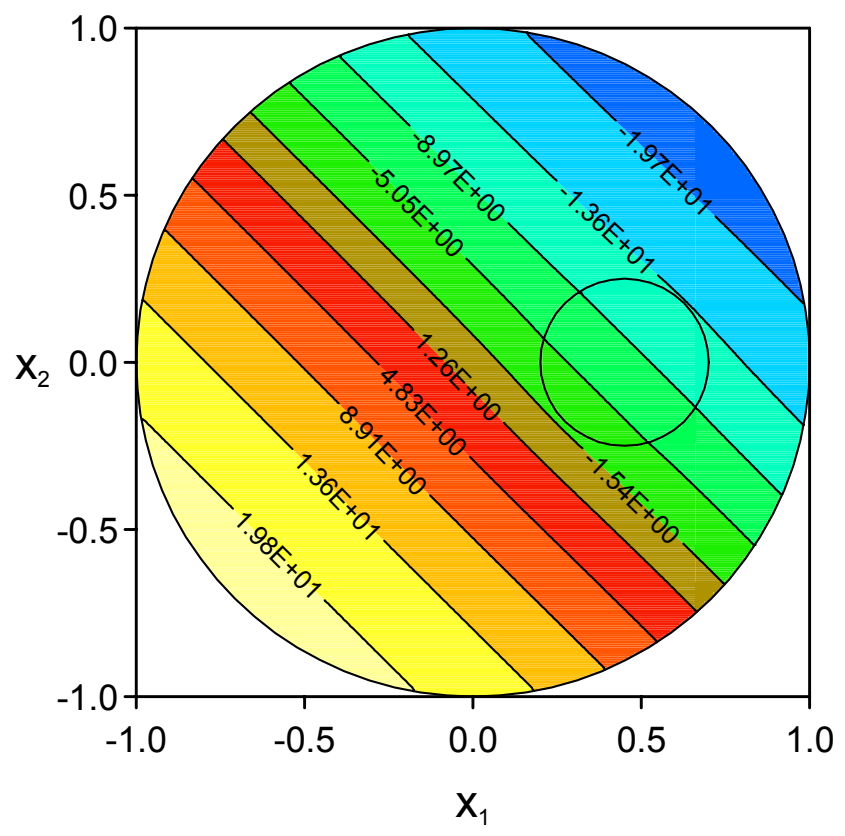

(b) Example 1: $v\left(t=0.2 \times 10^{-6} \mathrm{~s}\right)$

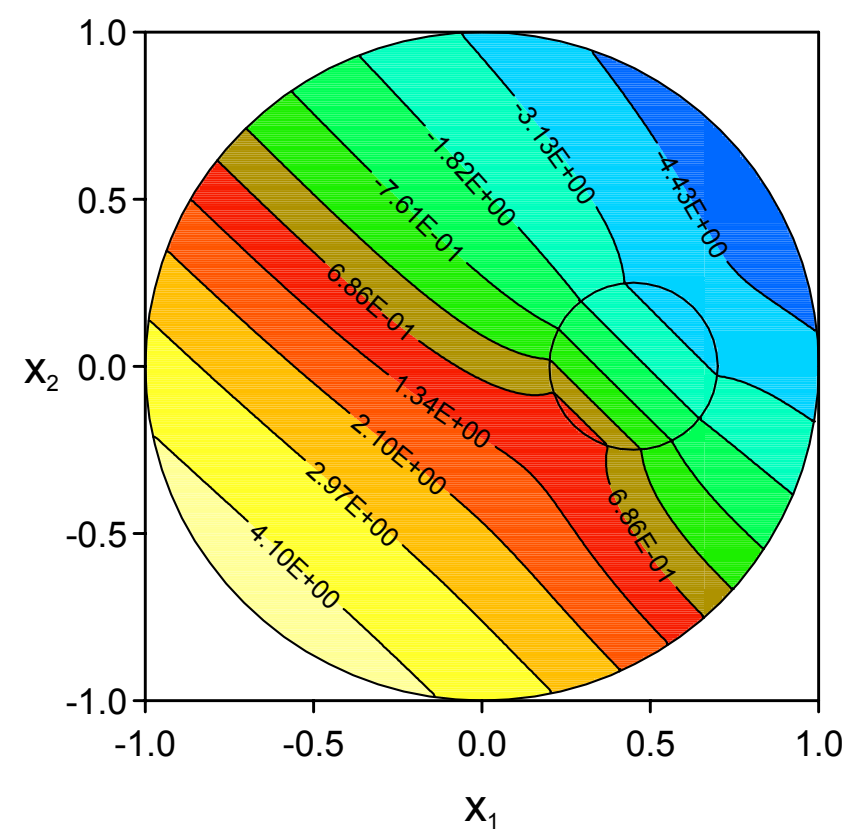

(c) Example 1: $v\left(t=0.5 \times 10^{-6} \mathrm{~s}\right)$

Figure 6: Distribution of the real part of the potential, $v=\operatorname{Re} \phi$, for $M=N=160$ at various times, namely (a) $t=0 \mathrm{~s}$, (b) $t=0.2 \times 10^{-6} \mathrm{~s}$ and (c) $t=0.5 \times 10^{-6} \mathrm{~s}$, for Example 1 . 


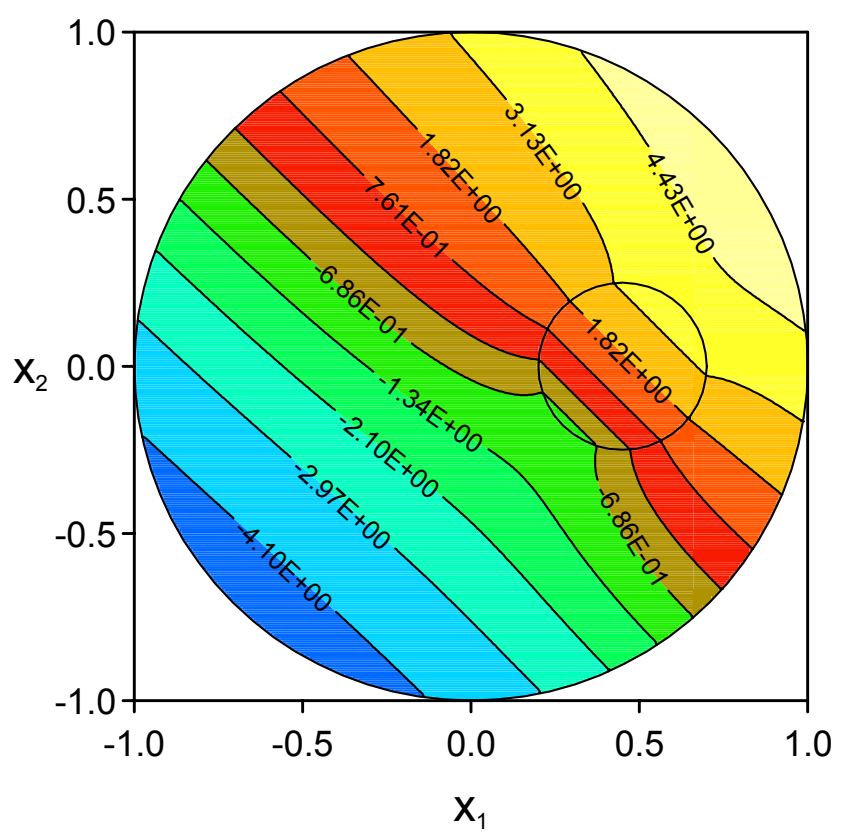

(a) Example 1: $h(t=0 \mathrm{~s})$

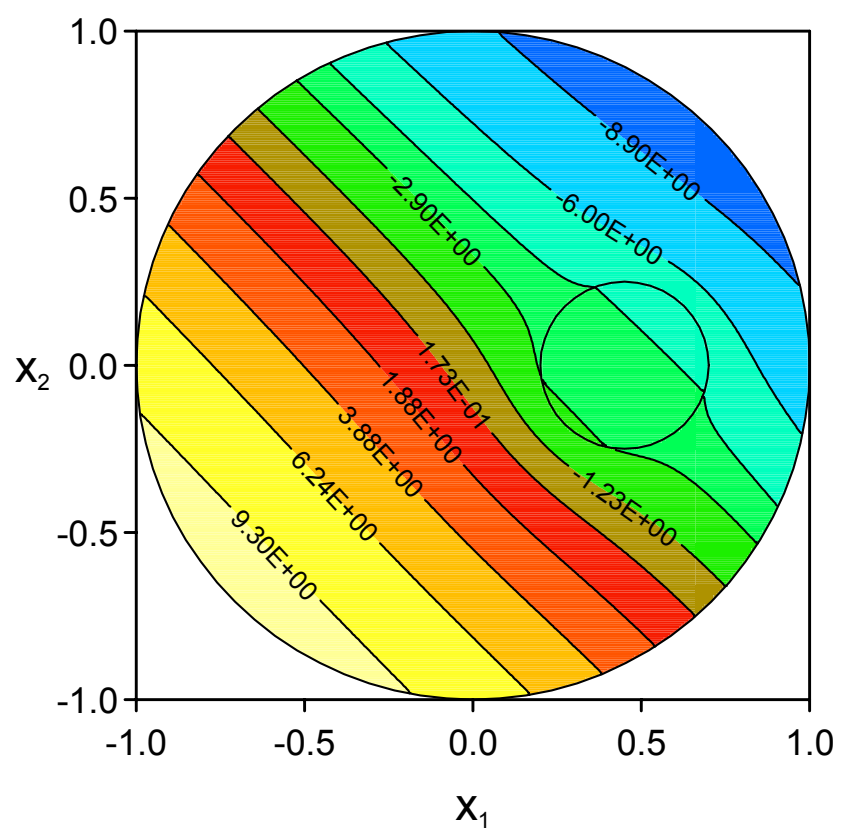

(b) Example 1: $h\left(t=0.2 \times 10^{-6} \mathrm{~s}\right)$

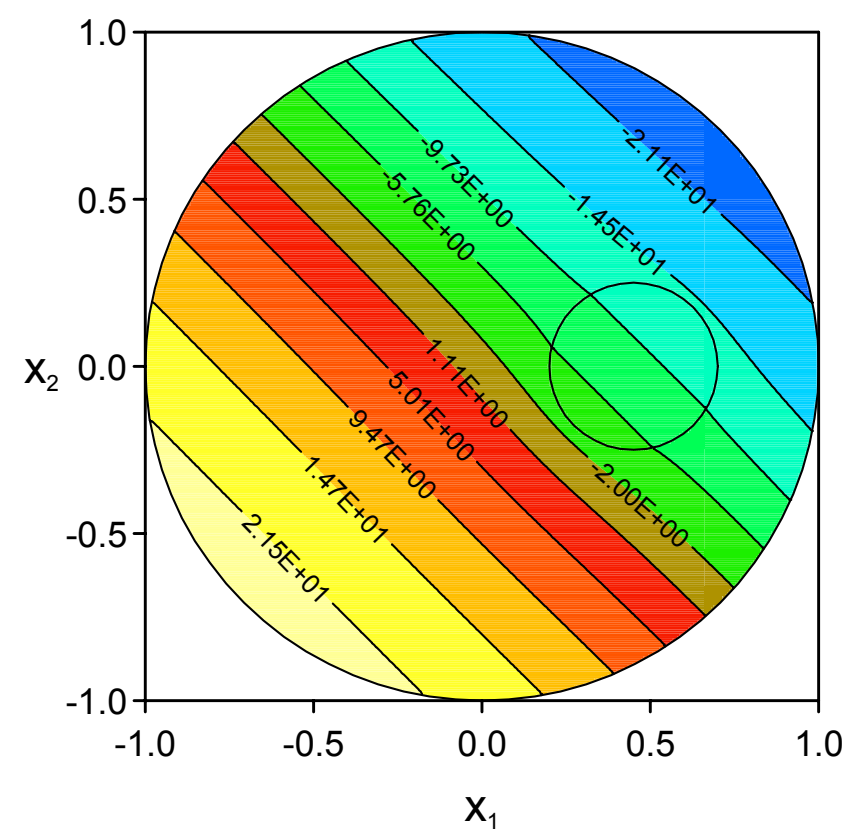

(c) Example 1: $h\left(t=0.5 \times 10^{-6} \mathrm{~s}\right)$

Figure 7: Distribution of the imaginary part of the potential, $h=\operatorname{Im} \phi$, for $\mathrm{M}=\mathrm{N}=160$ at various times, namely (a) $t=0 \mathrm{~s}$, (b) $t=0.2 \times 10^{-6} \mathrm{~s}$ and (c) $t=0.5 \times 10^{-6} \mathrm{~s}$, for Example 1 . 


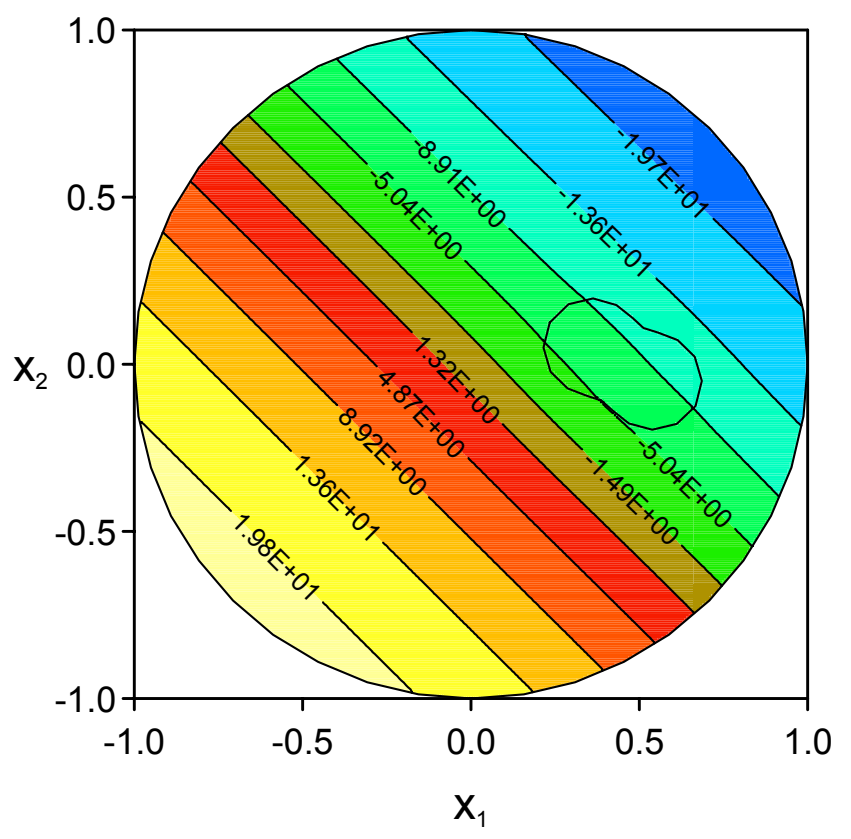

(a) Example 2: $v\left(t=0.2 \times 10^{-6} \mathrm{~s} ; \mathrm{M}=\mathrm{N}=80\right)$

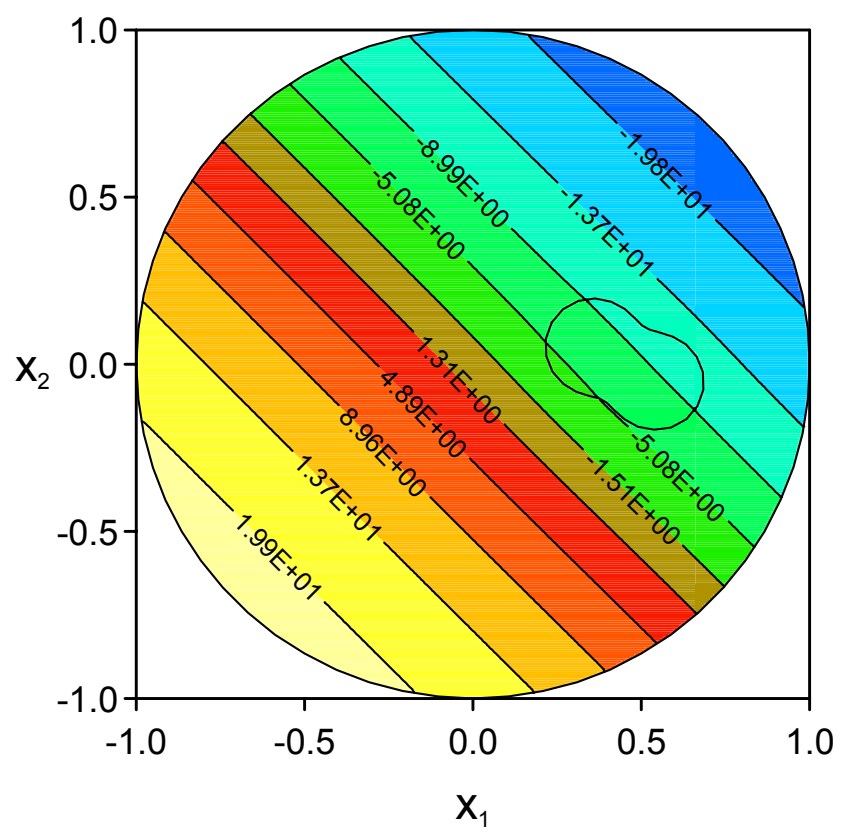

(b) Example 2: $v\left(t=0.2 \times 10^{-6} \mathrm{~s} ; \mathrm{M}=\mathrm{N}=120\right)$

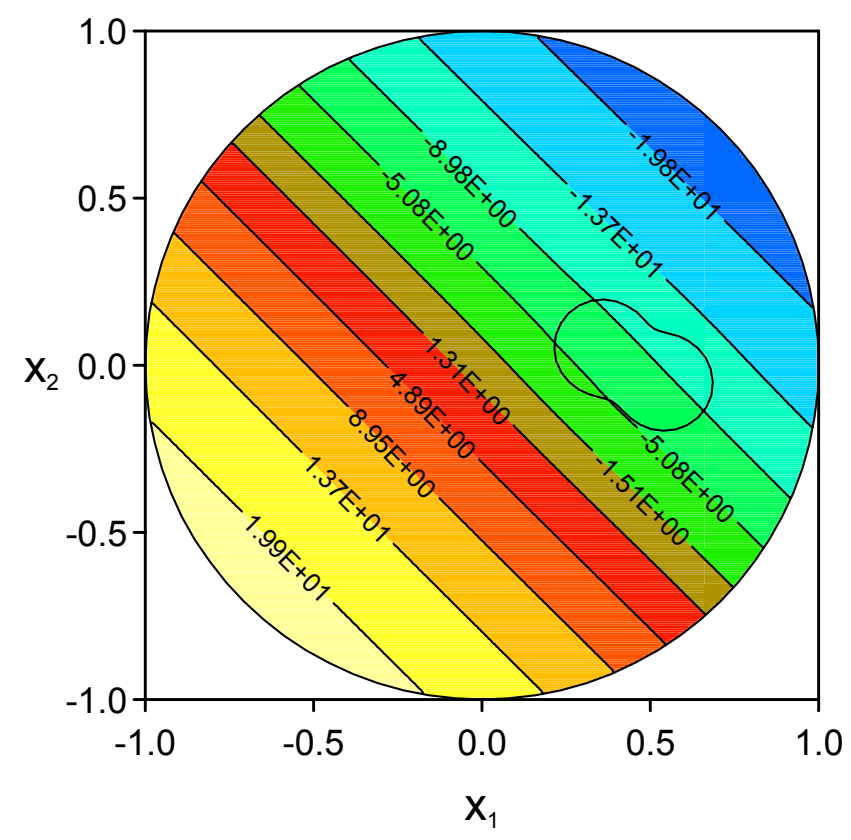

(c) Example 2: $v\left(t=0.2 \times 10^{-6} \mathrm{~s} ; \mathrm{M}=\mathrm{N}=160\right)$

Figure 8: Distribution of the real part of the potential, $v=\operatorname{Re} \phi$, at $t=0.2 \times 10^{-6} \mathrm{~s}$ and various numbers of collocation and source points, namely (a) $\mathrm{M}=\mathrm{N}=80$, (b) $\mathrm{M}=\mathrm{N}=120$ and (c) $\mathrm{M}=\mathrm{N}=160$, for Example 2. 


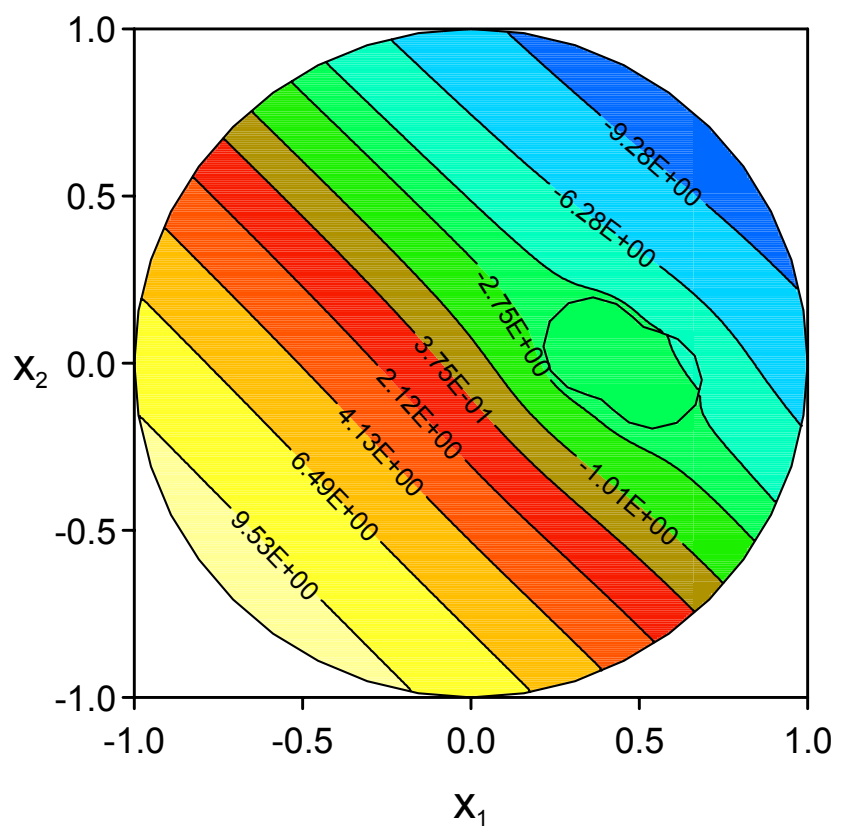

(a) Example 2: $h\left(t=0.2 \times 10^{-6} \mathrm{~s} ; \mathrm{M}=\mathrm{N}=80\right)$

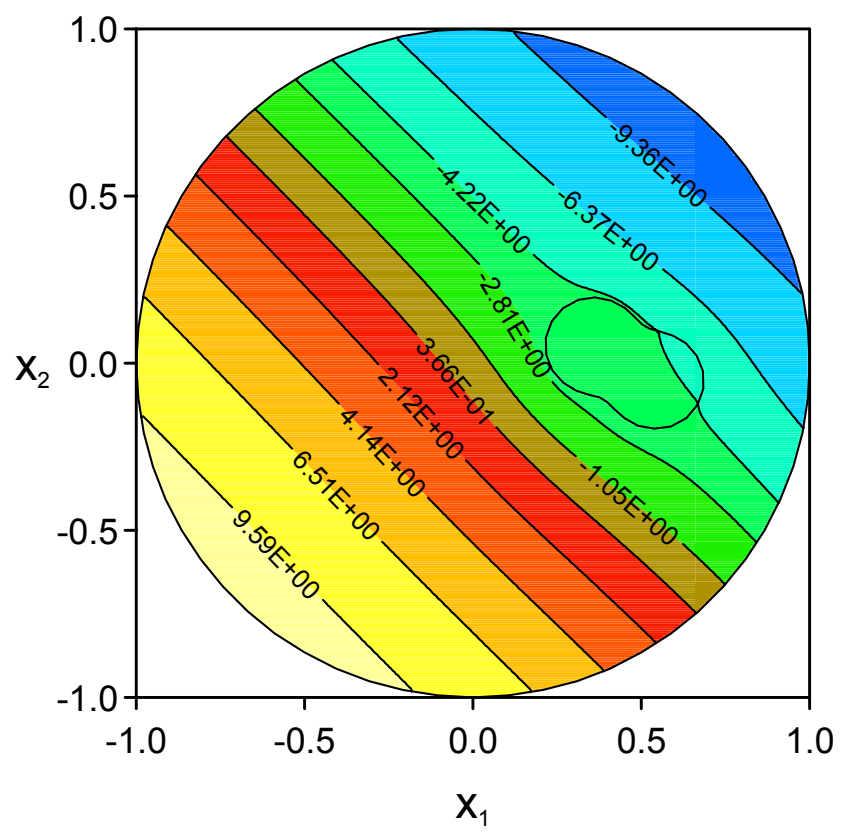

(b) Example 2: $h\left(t=0.2 \times 10^{-6} \mathrm{~s} ; \mathrm{M}=\mathrm{N}=120\right)$

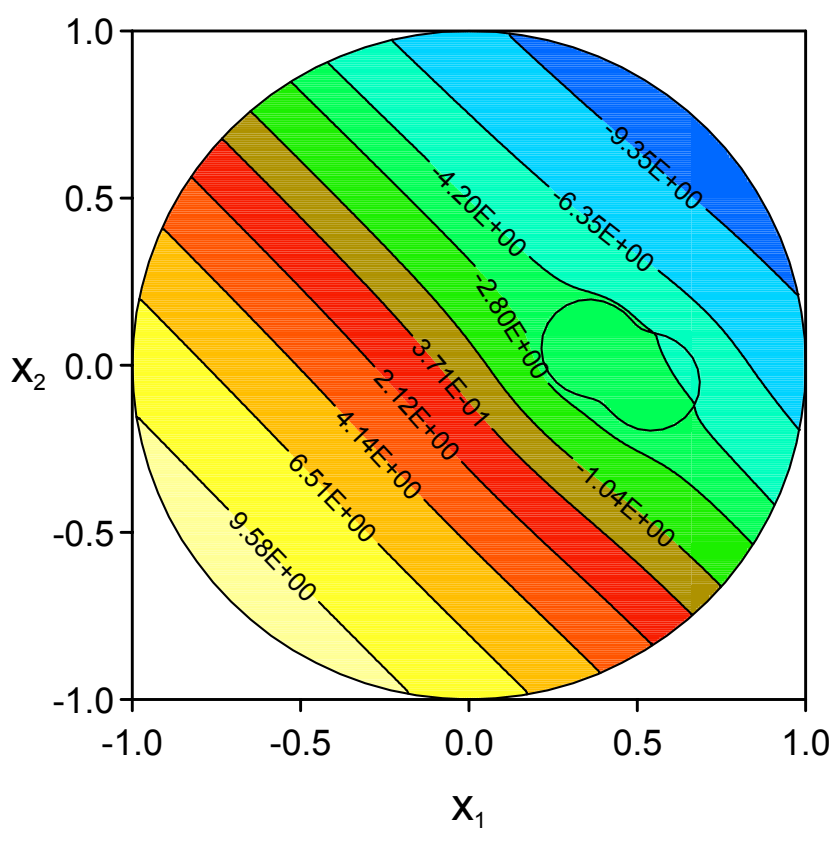

(c) Example 2: $h\left(t=0.2 \times 10^{-6} \mathrm{~s} ; \mathrm{M}=\mathrm{N}=160\right)$

Figure 9: Distribution of the imaginary part of the potential, $h=\operatorname{Im} \phi$, at $t=0.2 \times 10^{-6} \mathrm{~s}$ and various numbers of collocation and source points, namely (a) $\mathrm{M}=\mathrm{N}=80$, (b) $\mathrm{M}=\mathrm{N}=120$ and (c) $\mathrm{M}=\mathrm{N}=160$, for Example 2 . 


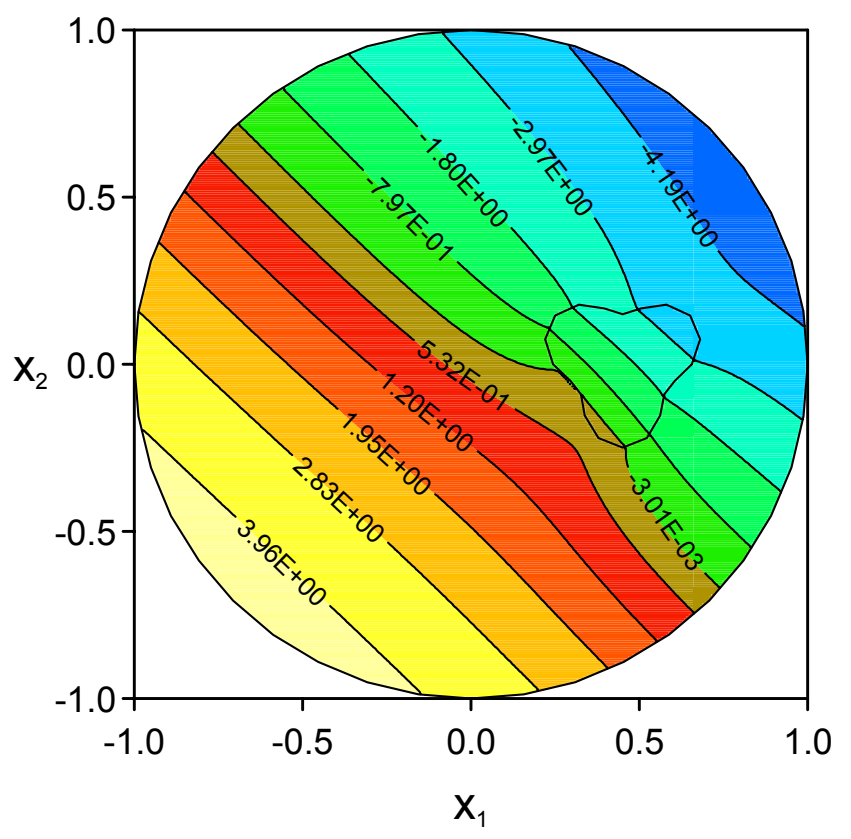

(a) Example 3: $v\left(t=0.5 \times 10^{-6} \mathrm{~s} ; \mathrm{M}=\mathrm{N}=80\right)$

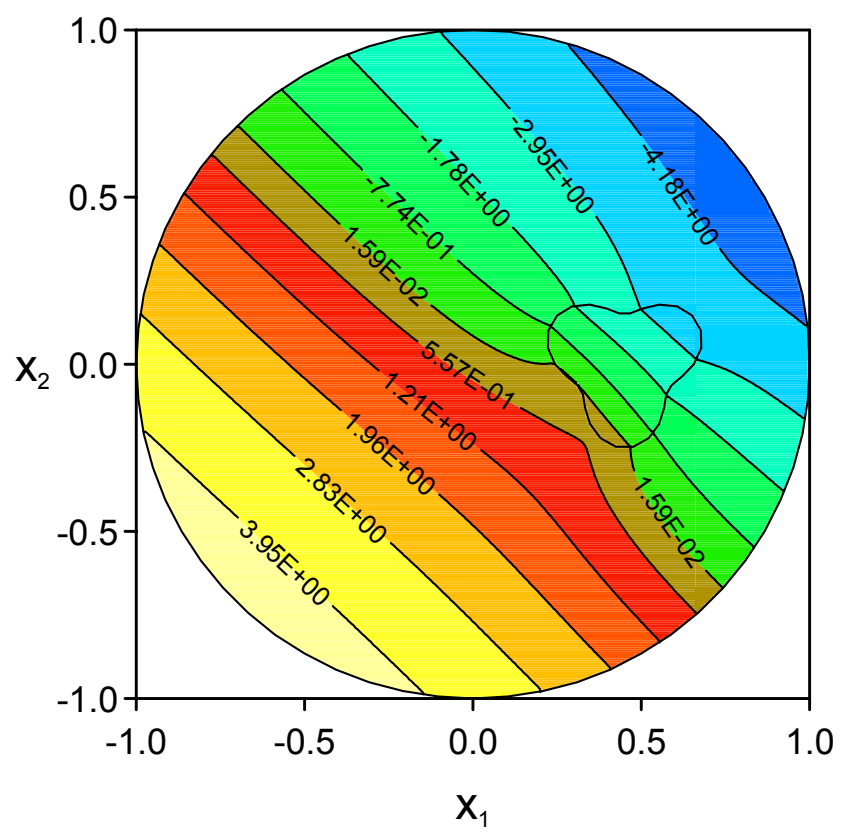

(b) Example 3: $v\left(t=0.5 \times 10^{-6} \mathrm{~s} ; \mathrm{M}=\mathrm{N}=120\right)$

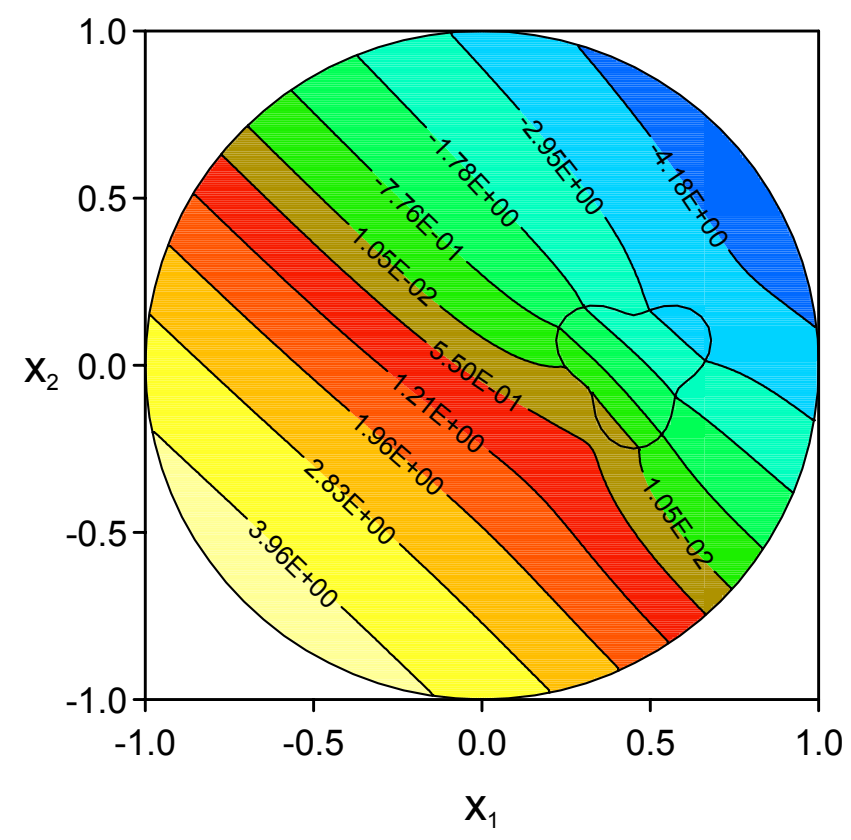

(c) Example 3: $v\left(t=0.5 \times 10^{-6} \mathrm{~s} ; \mathrm{M}=\mathrm{N}=160\right)$

Figure 10: Distribution of the real part of the potential, $v=\operatorname{Re} \phi$, at $t=0.5 \times 10^{-6} \mathrm{~s}$ and various numbers of collocation and source points, namely (a) $\mathrm{M}=\mathrm{N}=80$, (b) $\mathrm{M}=\mathrm{N}=120$ and (c) $\mathrm{M}=\mathrm{N}=$ 160, for Example 3. 


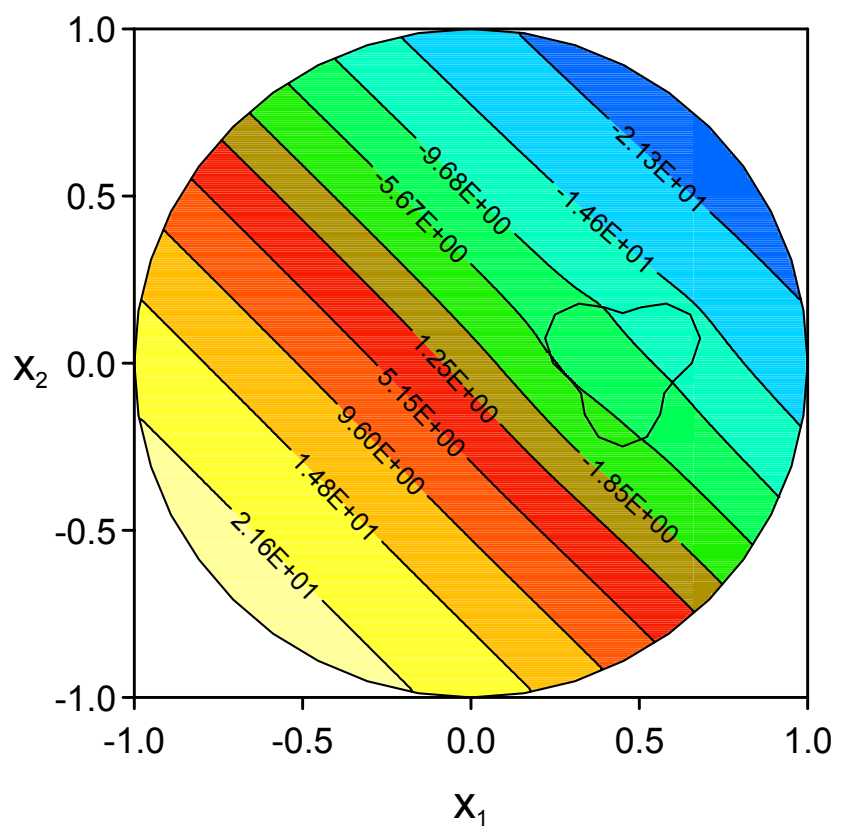

(a) Example 3: $h\left(t=0.5 \times 10^{-6} \mathrm{~s} ; \mathrm{M}=\mathrm{N}=80\right)$

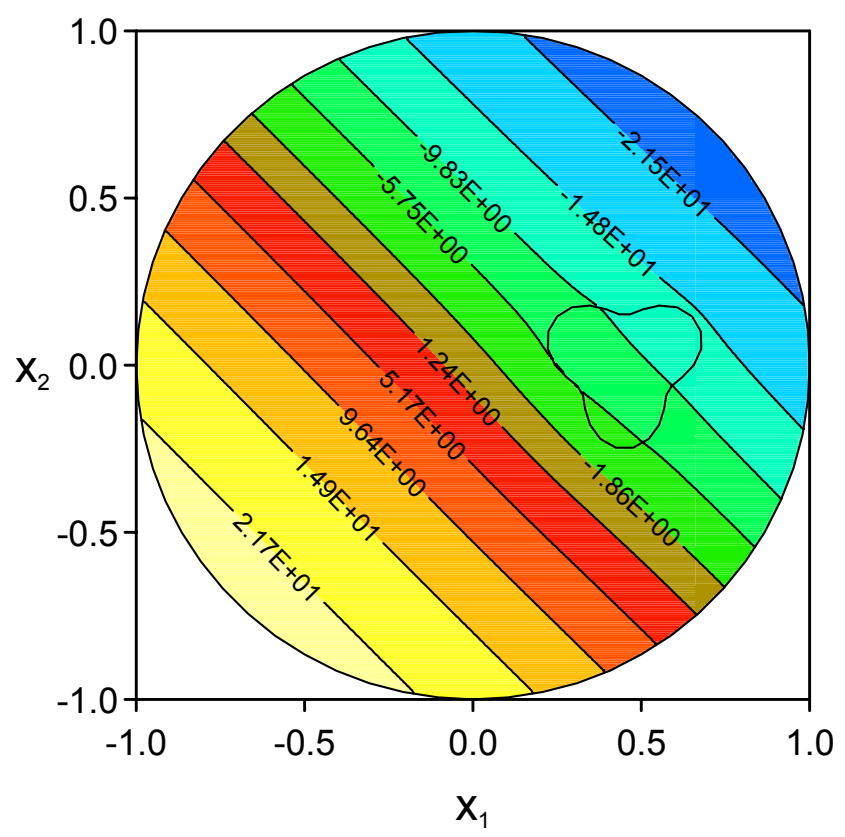

(b) Example 3: $h\left(t=0.5 \times 10^{-6} \mathrm{~s} ; \mathrm{M}=\mathrm{N}=120\right)$

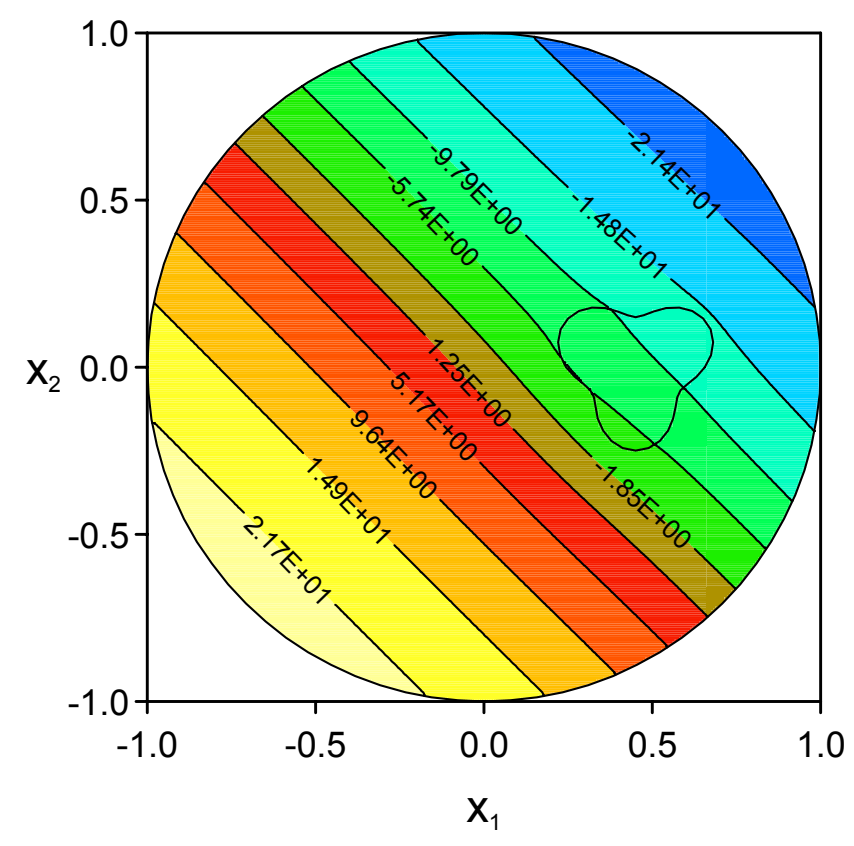

(c) Example 3: $h\left(t=0.5 \times 10^{-6} \mathrm{~s} ; \mathrm{M}=\mathrm{N}=160\right)$

Figure 11: Distribution of the imaginary part of the potential, $h=\operatorname{Im} \phi$, at $t=0.5 \times 10^{-6} \mathrm{~s}$ and various numbers of collocation and source points, namely (a) $\mathrm{M}=\mathrm{N}=80$, (b) $\mathrm{M}=\mathrm{N}=120$ and (c) $\mathrm{M}=\mathrm{N}=160$, for Example 3 . 


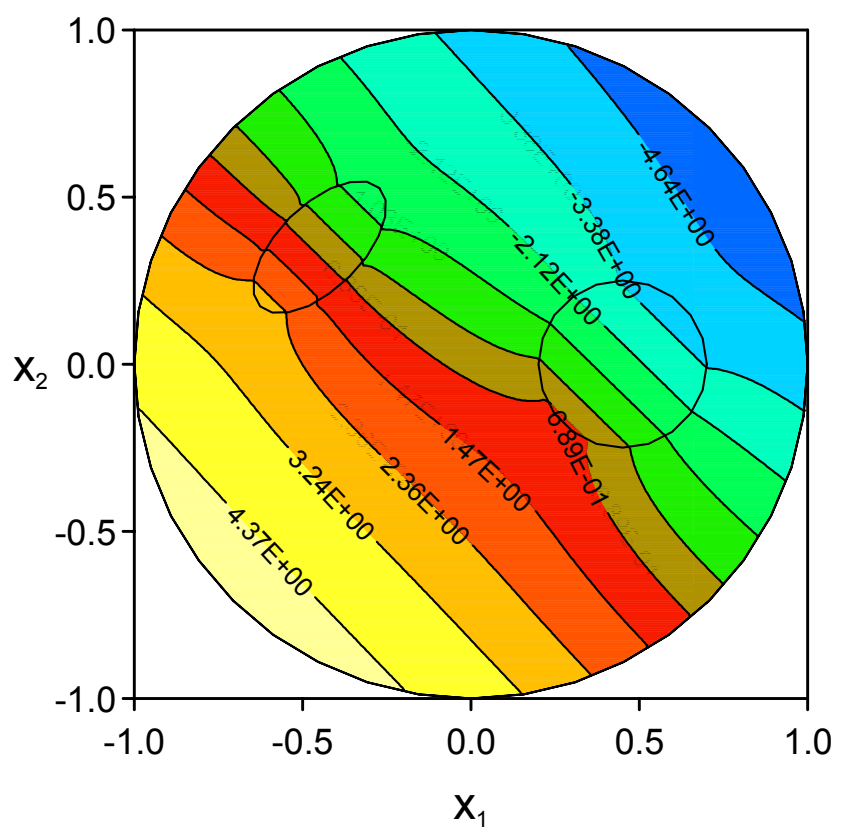

(a) Example 4: $v\left(t=0.5 \times 10^{-6} \mathrm{~s} ; \mathrm{M}=\mathrm{N}=120\right)$

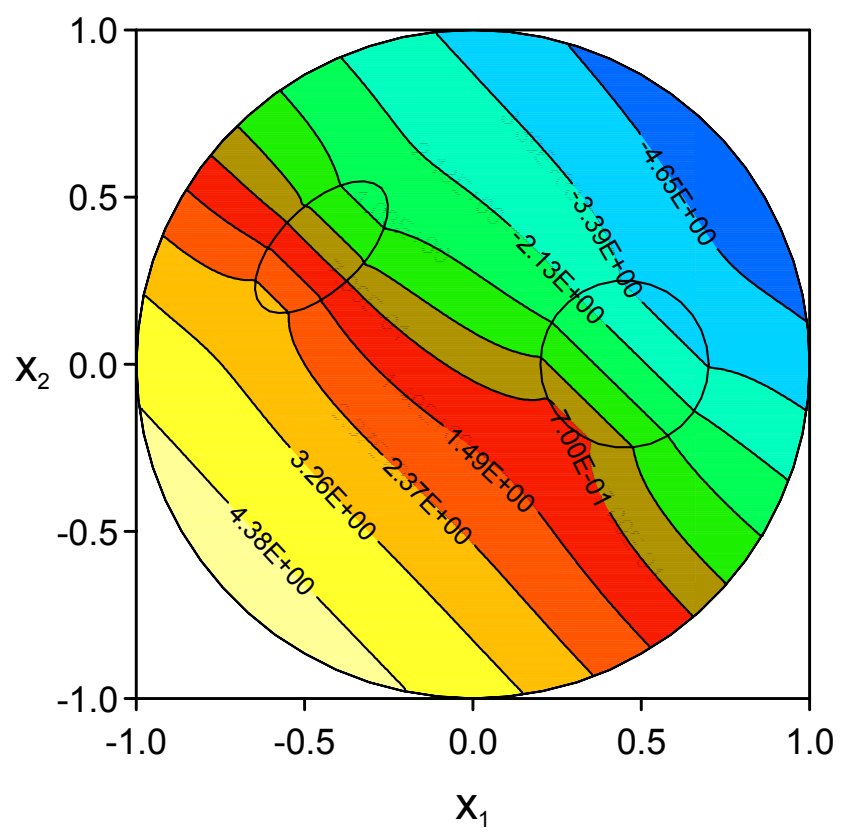

(b) Example 4: $v\left(t=0.5 \times 10^{-6} \mathrm{~s} ; \mathrm{M}=\mathrm{N}=180\right)$

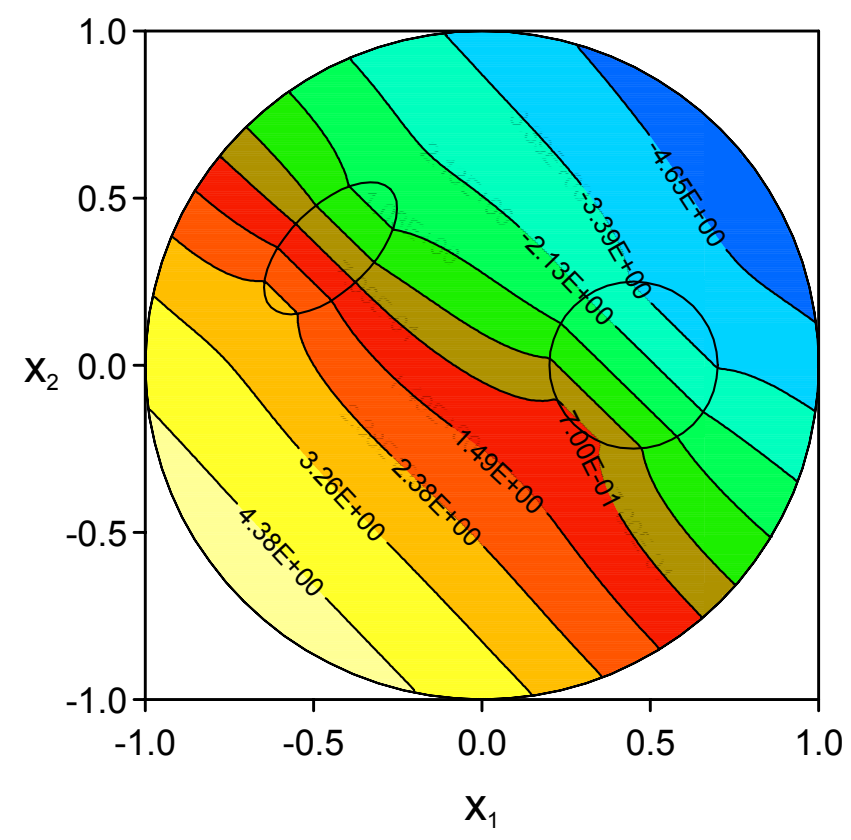

(c) Example 4: $v\left(t=0.5 \times 10^{-6} \mathrm{~s} ; \mathrm{M}=\mathrm{N}=240\right)$

Figure 12: Distribution of the real part of the potential, $v=\operatorname{Re} \phi$, at $t=0.5 \times 10^{-6} \mathrm{~s}$ and various numbers of collocation and source points, namely (a) $\mathrm{M}=\mathrm{N}=120$, (b) $\mathrm{M}=\mathrm{N}=180$ and (c) $\mathrm{M}=$ $\mathrm{N}=240$, for Example 4 . 


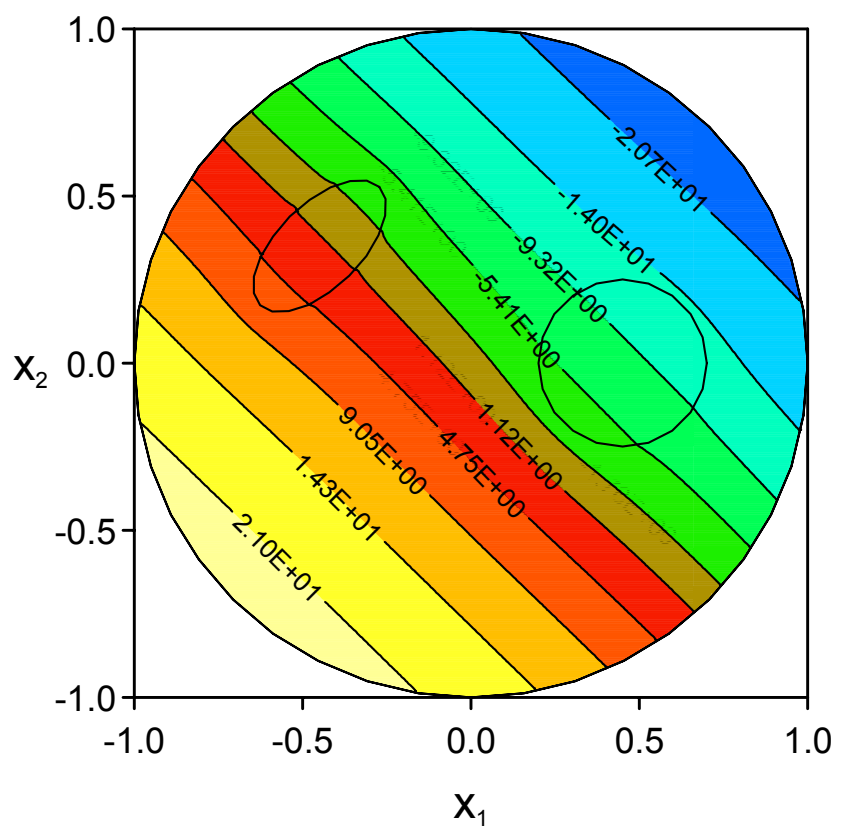

(a) Example 4: $h\left(t=0.5 \times 10^{-6} \mathrm{~s} ; \mathrm{M}=\mathrm{N}=120\right)$

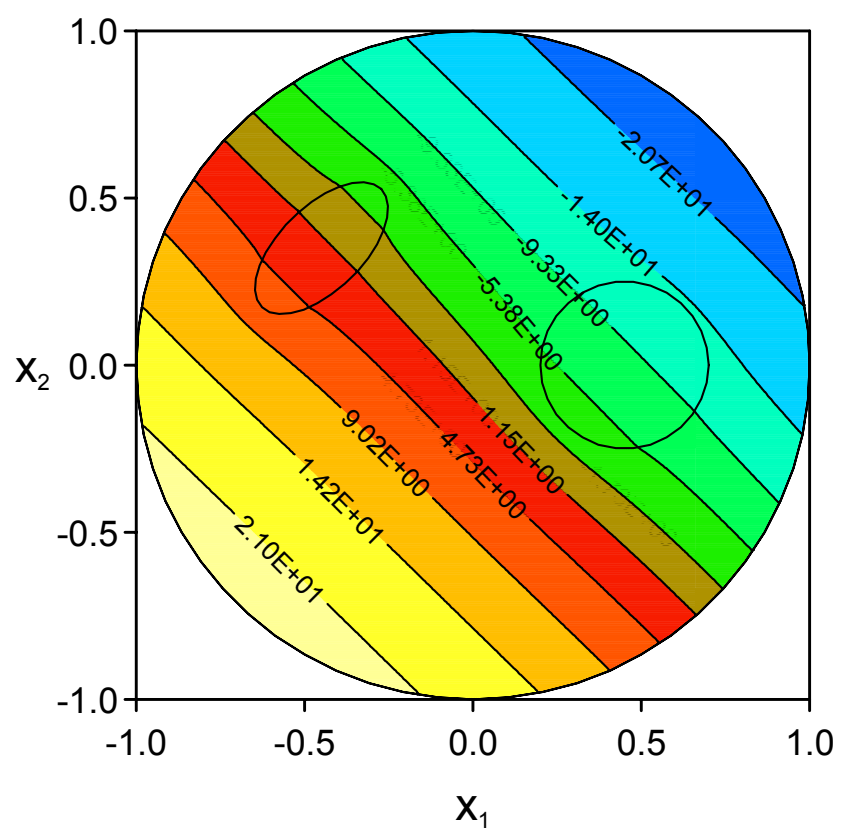

(b) Example 4: $h\left(t=0.5 \times 10^{-6} \mathrm{~s} ; \mathrm{M}=\mathrm{N}=180\right)$

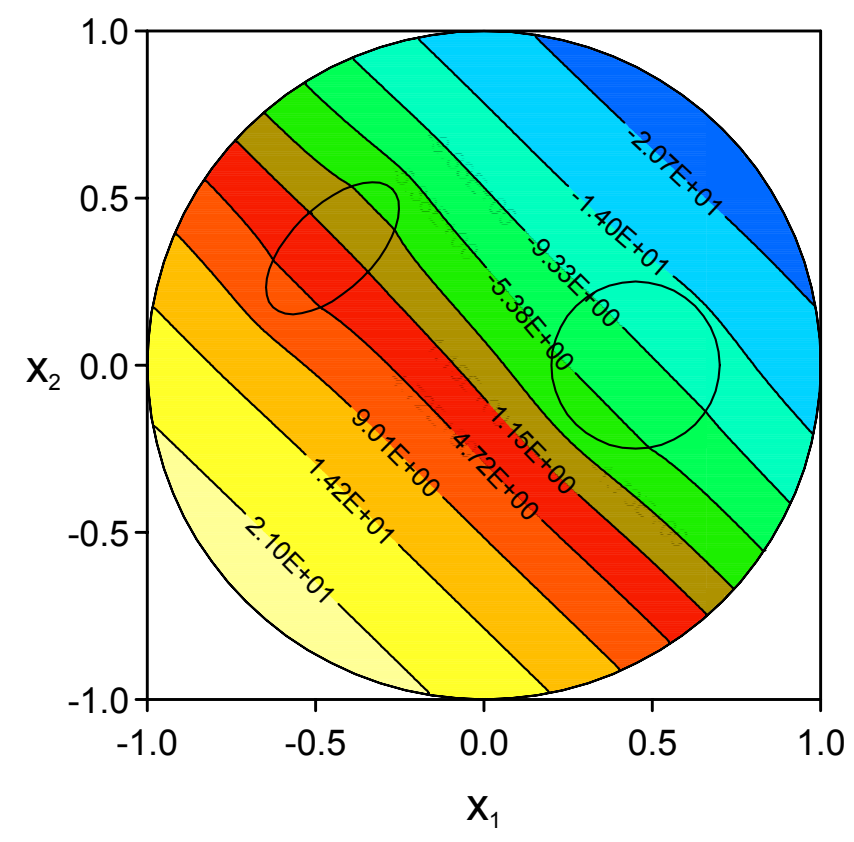

(c) Example 4: $h\left(t=0.5 \times 10^{-6} \mathrm{~s} ; \mathrm{M}=\mathrm{N}=240\right)$

Figure 13: Distribution of the imaginary part of the potential, $h=\operatorname{Im} \phi$, at $t=0.5 \times 10^{-6} \mathrm{~s}$ and various numbers of collocation and source points, namely (a) $\mathrm{M}=\mathrm{N}=120$, (b) $\mathrm{M}=\mathrm{N}=180$ and (c) $\mathrm{M}=\mathrm{N}=240$, for Example 4 . 